\title{
Discrete Boussinesq-type equations
}

\author{
Jarmo Hietarinta, ${ }^{1 *}$ and Da-jun Zhang ${ }^{2 \dagger}$ \\ ${ }^{1}$ Department of Physics and Astronomy, University of Turku, FIN-20014 Turku, Finland \\ ${ }^{2}$ Department of Mathematics, Shanghai University, Shanghai 200444, P.R. China
}

December 29, 2020

\begin{abstract}
We present a comprehensive review of the discrete Boussinesq equations based on their three-component forms on an elementary quadrilateral. These equations were originally found by Nijhoff et al using the direct linearization method and later generalized by Hietarinta using a search method based on multidimensional consistency. We derive from these three-component equations their two- and onecomponent variants. From the one-component form we derive two different semicontinuous limits as well as their fully continuous limits, which turn out to be PDE's for the regular, modified and Schwarzian Boussinesq equations. Several kinds of Lax pairs are also provided. Finally we give their Hirota bilinear forms and multi-soliton solutions in terms of Casoratians.
\end{abstract}

Keywords: Boussinesq equation, discrete lattice systems, continuum limits, Lax pairs, Hirota bilinear forms.

PACS numbers: 02.30.Ik, 02.90.+p

MSC: 39-04, 39A05, 39A14

\section{Contents}

1 Introduction 3

2 DBSQ-type equations 4

2.1 Basic concepts and definitions . . . . . . . . . . . . . . 4

2.2 Hietarinta's list of equations . . . . . . . . . . . . . . . . . . . . . . 5

2.3 Relations between the C-equations . . . . . . . . . . . . . . 6

2.4 Symmetries . . . . . . . . . . . . . . . . . . 8

2.4.1 $n \leftrightarrow m$ reflection symmetry . . . . . . . . . . . . 8

2.4 .2 Reversal symmetry . . . . . . . . . . . . . . . . 8

2.5 Initial values and evolution . . . . . . . . . . . . . . . . . . . . . . . 9

2.6 Connection with the direct-linearization results . . . . . . . . . . . 10

2.6.1 The direct-linearization approach . . . . . . . . . . . . . . 10

$2.6 .2 \mathrm{~B} 2 \ldots \ldots \ldots \ldots \ldots \ldots \ldots$

*E-mail: jarmo.hietarinta@utu.fi

${ }^{\dagger}$ E-mail: djzhang@staff.shu.edu.cn 


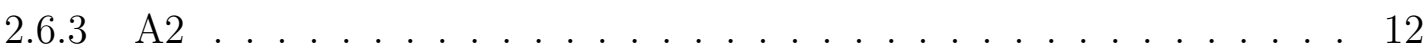

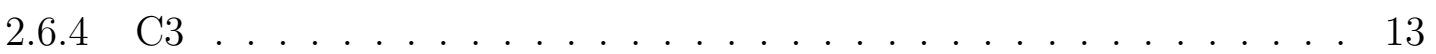

3 Two- and one-component forms 14

3.1 Generalities about the elimination process . . . . . . . . . 15

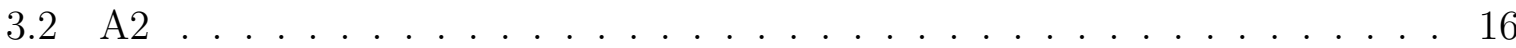

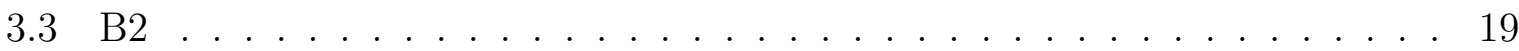

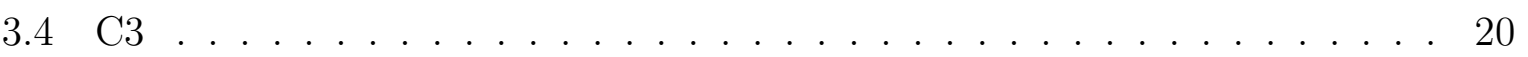

4 Continuum limits 22

4.1 Common features . . . . . . . . . . . . . . . . . . . 22

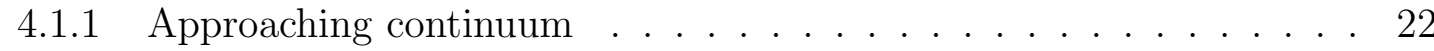

4.1.2 Parameter relations . . . . . . . . . . . . . . . . 23

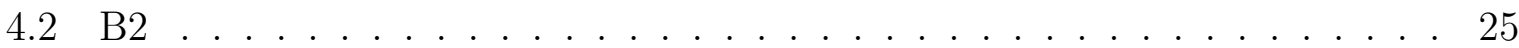

4.2 .1 Straight limit . . . . . . . . . . . . . . . 25

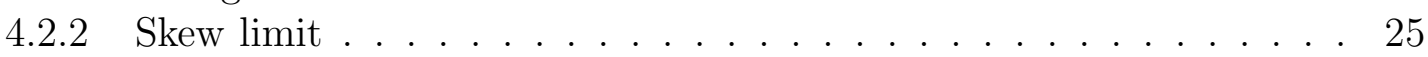

4.2 .3 Double limit . . . . . . . . . . . . . . . . . . . . . . . . . . . . . . . 25

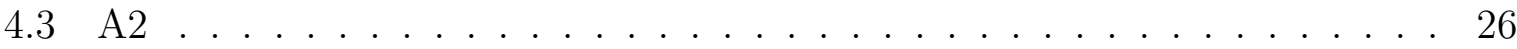

4.3.1 Straight limit . . . . . . . . . . . . . . 26

4.3 .2 Skew limit . . . . . . . . . . . . . . 27

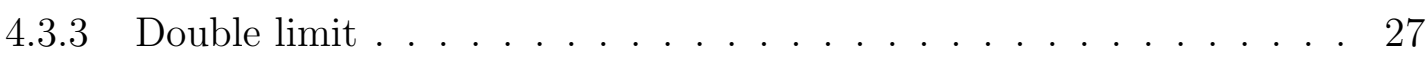

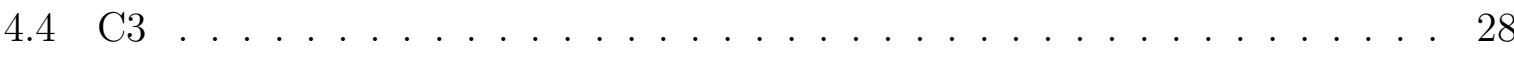

4.4 .1 Straight limit . . . . . . . . . . . . . . . 28

4.4 .2 Skew limit . . . . . . . . . . . . . . . . 28

4.4 .3 Double limit . . . . . . . . . . . . . . . . . . . . . . . 29

5 Lax pairs 29

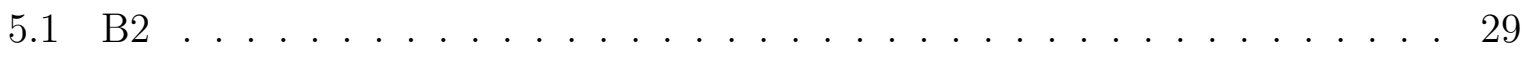

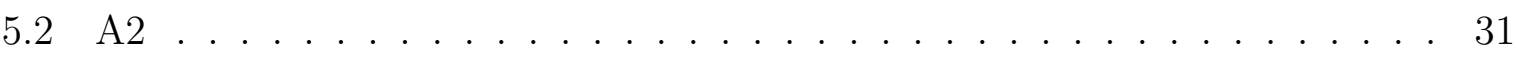

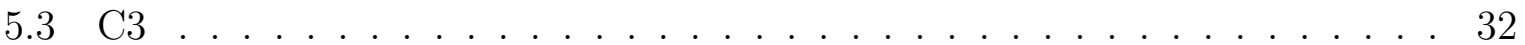

6 Bilinear structures of DBSQ-type equations 33

6.1 Preliminary . . . . . . . . . . . . . . . . . . 33

6.1.1 Discrete Hirota's bilinear form and Casoratians . . . . . . . . . . . 33

6.1 .2 Laplace expansion . . . . . . . . . . . . . . . . . 35

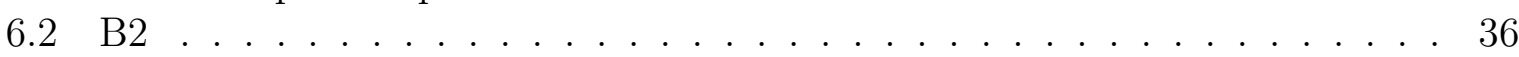

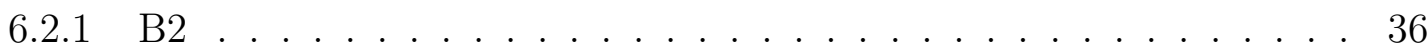

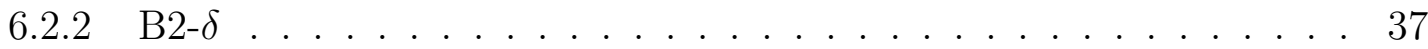

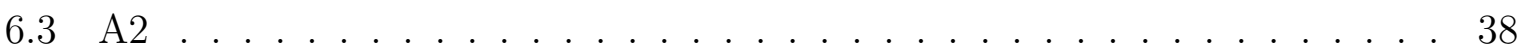

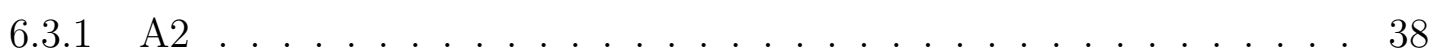

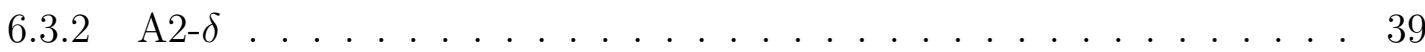

$6.4 \quad \mathrm{C} 3_{b_{0}} \ldots \ldots \ldots \ldots \ldots \ldots$

7 Conclusions 40

A Triply shifted variables from CAC 40

A.1 Three-component forms . . . . . . . . . . . . . . . 41

A.2 Two-component forms . . . . . . . . . . . . . . . . . . 42 


\section{Introduction}

Among the $1+1$ dimensional soliton equations there are evolution equations, such as the Korteweg - de Vries (KdV) equation, in which time derivatives appear in first order, but there are also important equations with higher order time derivatives, such as the Boussinesq (BSQ) equation. An essential difference between these equations is in the initial data required: For $\mathrm{KdV}$ it would be enough to give, e.g., $u(x, t=0)$, while for the second order BSQ equation we would need $u(x, t=0)$ and $\partial_{x} u(x, t=0)$, or something similar.

The difference between the first and second order time evolution is reflected also in the integrable discretizations of these equations. For first order equations a well defined evolution is obtained from a staircase-like initial data together with an equation defined on the elementary square of the lattice. For higher order time evolutions one would then need either initial data on a number of parallel staircases with an equation on a larger stencil or alternatively, multi-component initial data with a larger number of equations on the small stencil.

The recent rapid advances in the study of integrable partial difference equations $(\mathrm{P} \Delta \mathrm{E})$ are to a large extent due to the efficient use of the particular integrability property of multidimensional consistency (MDC), which is related to the existence of hierarchies in the continuous case [16]. In its simplest form it involves dimensions 2 and 3 and is called Consistency-Around-a-Cube (CAC). The MDC property was discussed already in [2, 25, 30, but in full force it was applied in [1] (with some further technical assumptions), and this provided a classification of first order equations defined on an elementary lattice square of the Cartesian 2D lattice, the so called Adler-Bobenko-Suris (ABS) list. The requirement of MDC can also be applied on multicomponent equations on the elementary plaquette. A partial classification of three-component equations was done in [14] on the basis of CAC and most of the results turned out to be discrete versions BSQ equations (DBSQ).

Multicomponent equations were also studied from the perspective of direct linearization approach (DLA) and several equations were found [11,27, 28, 33, 37, 41]. In addition to the CAC and DLA approaches DBSQ equations have been derived also by applying a three-reduction on the three-term Hirota-Miwa equation [6, 22], or on the four-term Miwa equation [20]. Still further results have been obtained using the Cauchy matrix approach [7] or graded Lax pairs [9].

In this review we will discuss in detail the multi-component DBSQ equations. In Section 2 we compare the various three-component forms that have appeared in the literature and their connections by gauge transformations or by Möbius transformations. Their symmetries are also briefly discussed. In fact, all the DBSQ-type equations found in [14] can be viewed as extensions of some known lattice equations found in 1990's. In Section 3 we discuss how the dynamics of the first order three-component equations can be represented by two- or one-component forms on a larger stencil. We also discuss the continuum limits of the one-component forms in Section 4 and show that the limits really are BSQ-like equations.

In Section 5] we present the Lax pairs of the various discrete forms. As for the solutions of DBSQ-type equations, besides the results from direct linearisation and Cauchy matrix approach (see [7, 27, 36, 37, 41]), equation B2 has been bilinearized in [18] and solutions were given in terms of Casoratians; in Section 6 we investigate bilinear forms for A2 and C3 equations. 


\section{DBSQ-type equations}

\subsection{Basic concepts and definitions}

The discrete equations that we discuss here are all defined on the Cartesian $\mathbb{Z} \times \mathbb{Z}$ lattice. Most of the time the equations are defined on a single quadrilateral but larger stencils are sometimes needed. The independent variables live on the vertices of the lattice and are therefore labeled by the vertex coordinates, see Figure 1 .

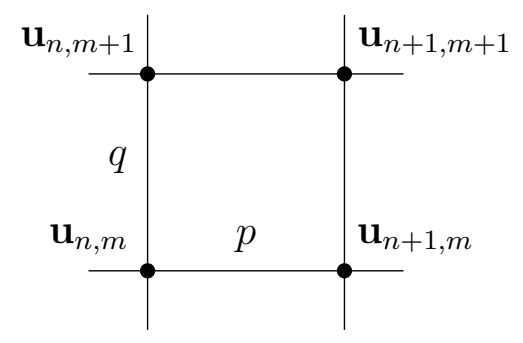

Figure 1: Elementary quadrilateral of the lattice, with possibly multi-component corner variables $\mathbf{u}$. The parameters $p, q$ characterize the distance between lattice points.

Sometimes the equations have a simpler look if we replace the subscript with a tilde or a hat, or use some other simplified notation, for example

$$
\mathbf{u}=\mathbf{u}_{n, m}=\mathbf{u}_{0,0}, \widetilde{\mathbf{u}}=\mathbf{u}_{n+1, m}=\mathbf{u}_{1,0}, \widehat{\mathbf{u}}=\mathbf{u}_{n, m+1}=\mathbf{u}_{0,1}, \widehat{\widetilde{\mathbf{u}}}=\mathbf{u}_{n+1, m+1}=\mathbf{u}_{1,1}
$$

The equation(s) on the quadrilateral are given by $\mathbf{Q}(\mathbf{u}, \widetilde{\mathbf{u}}, \widehat{\mathbf{u}}, \widehat{\widetilde{\mathbf{u}}})=0$, where $\mathbf{Q}$ are affine multilinear polynomials, and one may then ask whether the system of equations is integrable according to some definition. We will use the MDC criterion which means that the equation defined on the 2D-lattice can be extended consistently into higher dimensions.

As an example consider the simple case of the 1-component lattice potential KdV equation (H1 in the ABS list):

$$
Q(u, \widetilde{u}, \widehat{u}, \widehat{\widetilde{u}} ; p, q):=(u-\widehat{\widetilde{u}})(\widehat{u}-\widetilde{u})-p^{2}+q^{2}=0 .
$$

Since $u$ is defined on the 2D-lattice it naturally depends only on the coordinates $n, m$, but in order to extend the equations into 3D setting, which is the simplest requirement of MDC, we introduce a third variable $k$ and denote $u_{n, m, k+1}=\bar{u}$; the associated lattice parameter is $r$. For each of the six sides of the cube we have an equation:

$$
\begin{aligned}
& \text { bottom: } \quad Q(u, \widetilde{u}, \widehat{u}, \widehat{\widetilde{u}} ; p, q)=0, \quad \text { top: } \quad Q(\bar{u}, \overline{\widetilde{u}}, \overline{\widehat{u}}, \overline{\widehat{\widetilde{u}}} ; p, q)=0, \\
& \text { back: } Q(u, \widehat{u}, \bar{u}, \overline{\widehat{u}} ; \alpha, \beta)=0, \quad \text { front: } Q(\widetilde{u}, \widehat{\widetilde{u}}, \overline{\widetilde{u}}, \overline{\widehat{u}} ; \alpha, \beta)=0 \text {, } \\
& \text { left: } \quad Q(u, \bar{u}, \widetilde{u}, \overline{\widetilde{u}} ; \gamma, \delta)=0, \quad \text { right: } \quad Q(\widehat{u}, \overline{\widehat{u}}, \widehat{\widetilde{u}}, \overline{\widehat{u}} ; \gamma, \delta)=0 .
\end{aligned}
$$

(Note that we used arbitrary parameters in this example and hope to determine them by consistency.) In order to study CAC we choose $u, \widetilde{u}, \widehat{u}, \bar{u}$ as initial values and then from the equations on the LHS we can compute $\widehat{\widetilde{u}}, \overline{\widehat{u}}, \overline{\widetilde{u}}$. When these are used on the RHS we have three ways to compute $\overline{\widehat{u}}$ but they must all yield the same value. In this case we find the condition

$$
\left(\alpha^{2}-\beta^{2}\right)+\left(\gamma^{2}-\delta^{2}\right)+\left(p^{2}-q^{2}\right)=0 .
$$


Since back and front equations do not depend on $p$ we find $\left(\alpha^{2}-\beta^{2}\right)=\left(q^{2}-r^{2}\right)$ and $\left(\gamma^{2}-\delta^{2}\right)=r^{2}-p^{2}$ for some $r$ and then all RHS equations yield the very symmetric form

$$
\overline{\widehat{\widetilde{u}}}=\frac{\widehat{u} \widetilde{u}\left(q^{2}-p^{2}\right)+\widetilde{u} \bar{u}\left(p^{2}-r^{2}\right)+\bar{u} \widehat{u}\left(r^{2}-q^{2}\right)}{\bar{u}\left(p^{2}-q^{2}\right)+\widehat{u}\left(r^{2}-p^{2}\right)+\widetilde{u}\left(q^{2}-r^{2}\right)} .
$$

Note that the RHS of this expression does not depend on $u$, this is called the tetrahedron property.

The application of MDC on one-component equations resulted in the ABS-list [1]. For multi-component equations there are no equally comprehensive classifications. It should also be noted that passing the CAC test is necessary but not sufficient [15] for integrability.

\subsection{Hietarinta's list of equations}

In [14] Hietarinta made a partial classification of Boussinesq (BSQ) type lattice equations using CAC. Since BSQ equations are of second order in time, their discrete analogues are either multi-component on a quadrilateral, or defined on a larger stencil. In [14] threecomponent approach was used and some equations were defined on the links and one equation on the full quadrilateral. Using CAC, the following three-component DBSQtype equation were found:

B2:

$$
\begin{aligned}
& \widetilde{y}=x \widetilde{x}-z, \\
& \widehat{y}=x \widehat{x}-z, \\
& y=x \widehat{\widetilde{x}}-\widehat{\widetilde{z}}+b_{0}(\widehat{\widetilde{x}}-x)+b_{1}+\frac{P-Q}{\widetilde{x}-\widehat{x}}
\end{aligned}
$$

A2:

$$
\begin{aligned}
& \widetilde{y}=z \widetilde{x}-x, \\
& \widehat{y}=z \widehat{x}-x, \\
& y=x \widehat{\widetilde{z}}-b_{0} x+\frac{P \widetilde{x}-Q \widehat{x}}{\widehat{z}-\widetilde{z}},
\end{aligned}
$$

C3:

$$
\begin{aligned}
& \widetilde{y} z=\widetilde{x}-x, \\
& \widehat{y} z=\widehat{x}-x \\
& \widehat{\widetilde{z}} y=b_{0} x+b_{1}+z \frac{P \widetilde{y} \widehat{z}-Q \widehat{y} \widetilde{z}}{\widetilde{z}-\widehat{z}}
\end{aligned}
$$

and

C4:

$$
\begin{aligned}
& \widetilde{y} z=\widetilde{x}-x, \\
& \widehat{y} z=\widehat{x}-x, \\
& \widehat{\widetilde{z}} y=x \widehat{\widetilde{x}}+b_{2}+z \frac{P \widetilde{y} \widehat{z}-Q \widehat{y} \widetilde{z}}{\widetilde{z}-\widehat{z}} .
\end{aligned}
$$

Here the parameters $P$ and $Q$ are related to lattice spacing parameters $p, q$ in $n$ and $m$-directions, respectively. 
The convention for naming the variables was designed for MDC and for analyzing the evolution (Section 2.5): The quasilinear equations are defined on the edges of the quadrilateral; "a" equations depend always on $x, \widetilde{x}, z$, and $\widetilde{y}$, and the "b" equations on $x$, $\widehat{x}, z$, and $\widehat{y}$, i.e., the dependence is always the same, only the algebra is different. Also, we list the equations in the order B2, A2, C3,4 because, as we will see later, they correspond to regular, modified and Schwarzian BSQ equations, respectively.

The coupling constants $b_{i}$ are arbitrary and generalize some previous results. Note however, that $b_{1}$ in $\mathrm{B} 2$ can be removed with the transformation

$$
(x, y, z) \mapsto\left(x, y-\frac{b_{1}}{3}(n+m-1), z+\frac{b_{1}}{3}(n+m)\right),
$$

and $b_{0}$ in $\mathrm{A} 2$ can be removed using

$$
(x, y, z) \mapsto\left(x, y+\frac{b_{0} x}{3}(n+m), z+\frac{b_{0}}{3}(n+m+1)\right) .
$$

In the following we do not keep these removable parameters.

In addition some two-component forms were found in [14]:

C2-1:

$$
\begin{aligned}
& \widehat{\widetilde{x}}=\frac{\widehat{x} \widetilde{z}-\widetilde{x} \widehat{z}}{\widetilde{z}-\widehat{z}}, \\
& \widehat{\widetilde{z}}=-b_{0} z \widehat{\widetilde{x}}+z \frac{P \widehat{z}-Q \widetilde{z}}{\widetilde{z}-\widehat{z}},
\end{aligned}
$$

and

C2-2:

$$
\begin{aligned}
& \widehat{\widetilde{x}}=\frac{\widehat{x} \widetilde{z}-\widetilde{x} \widehat{z}}{\widetilde{z}-\widehat{z}}, \\
& x \widehat{\widetilde{z}}=-b_{0} z+z \frac{P \widetilde{x} \widehat{z}-Q \widehat{x} \widetilde{z}}{\widetilde{z}-\widehat{z}} .
\end{aligned}
$$

These are actually discrete versions of $\mathrm{KdV}$ so we will not discuss them further here.

\subsection{Relations between the C-equations}

Let us first note that lattice equations are classified only up to local rational-linear (i.e., Möbius) transformations, and that equations related by them are considered same. However, for some purposes a particular form may be better in practice.

Note that for the C-equations one can derive relation (2.9a) by eliminating $y$. After the transformations discussed below it is often useful to use this relation when comparing results.

First note that if $b_{0} \neq 0$ then by the transformation

$$
x \rightarrow x-\frac{b_{1}}{b_{0}}
$$

one can remove from the $\mathrm{C} 3$ equation (2.5c) the parameter $b_{1}$ and then we can consider the following form:

$$
\begin{aligned}
& \widetilde{y} z=\widetilde{x}-x, \\
& \widehat{y} z=\widehat{x}-x, \\
& \widehat{\widetilde{z}} y=b_{0} x+z \frac{P \widetilde{y} \widehat{z}-Q \widehat{y} \widetilde{z}}{\widetilde{z}-\widehat{z}},
\end{aligned}
$$


which we call $\mathrm{C} 3_{b_{0}}$.

Since the transformation (2.11) fails when $b_{0}=0$, the following equation

$$
\begin{aligned}
& \widetilde{y} z=\widetilde{x}-x, \\
& \widehat{y} z=\widehat{x}-x, \\
& \widehat{\widetilde{z}} y=b_{1}+z \frac{P \widetilde{y} \widehat{z}-Q \widehat{y} \widetilde{z}}{\widetilde{z}-\widehat{z}}
\end{aligned}
$$

is not a trivial subcase of C3 equation (2.5). Since (2.13c) does not contain $x$, we get a two-component form after eliminating $x$ from (2.13a, 2.13b) and their shifts, this results in

$$
\widehat{\widetilde{y}}=-z \frac{\widetilde{y}-\widehat{y}}{\widetilde{z}-\widehat{z}} .
$$

Thus (2.13c 2.13d) is a two component form, let us call it $\mathrm{C} 3_{b_{1}}$.

We will next show that $\mathrm{C} 4$ can be obtained from C3 by a Möbius transformation. As the first step we note that from equations (2.5a,2.5b) and their shifts one can derive

$$
x=\widehat{\widetilde{x}}+z \frac{\widetilde{y} \widehat{z}-\widehat{y} \widetilde{z}}{\widetilde{z}-\widehat{z}} .
$$

Using it to replace $\frac{1}{2} b_{0} x$ in (2.12c) we get the following alternative form for $\mathrm{C} 3_{b_{0}}$ :

$$
\begin{aligned}
& \widetilde{y} z=\widetilde{x}-x, \\
& \widehat{y} z=\widehat{x}-x, \\
& \widehat{\widetilde{z}} y=z \frac{\left(P-c_{2}\right) \widetilde{y} \widehat{z}-\left(Q-c_{2}\right) \widehat{y} \widetilde{z}}{\widetilde{z}-\widehat{z}}+c_{2}(x+\widehat{\widetilde{x}}), \quad\left(c_{2}=\frac{b_{0}}{2}\right) .
\end{aligned}
$$

Now, inserting the (mixed) Möbius transformation [41]

$$
x=\frac{x_{1}-c_{2}}{2 c_{2}\left(x_{1}+c_{2}\right)}, \quad y=\frac{y_{1}}{x_{1}+c_{2}}, \quad z=\frac{z_{1}}{x_{1}+c_{2}},
$$

into (2.15) we get

$$
\begin{aligned}
& \widetilde{y}_{1} z_{1}=\widetilde{x}_{1}-x_{1}, \\
& \widehat{y}_{1} z_{1}=\widehat{x}_{1}-x_{1}, \\
& \widehat{\widetilde{z}}_{1} y_{1}=z_{1} \frac{\left(P-c_{2}\right) \widetilde{y}_{1} \widehat{z}_{1}-\left(Q-c_{2}\right) \widehat{y}_{1} \widetilde{z}_{1}}{\widetilde{z}_{1}-\widehat{z}_{1}}+x_{1} \widehat{\widetilde{x}}_{1}-c_{2}^{2},
\end{aligned}
$$

which is $\mathrm{C}_{b_{2}}$ equation (2.6), after redefining

$$
P \rightarrow P-c_{2}, \quad Q \rightarrow Q-c_{2}, \quad b_{2}=-c_{2}^{2} .
$$

The above transformation fails if $b_{0}=0$ in C3 i.e., if $b_{2}=0$ in C4, but that special case can be obtained from $\mathrm{C} 3_{b_{1}=1}$ by the following transformation:

$$
x=-1 / x_{1}, \quad y=y_{1} / x_{1}, \quad z=z_{1} / x_{1} .
$$

But since $\mathrm{C} 3_{b_{1}=1}$ depends only on 2 variables, the transformation (2.19) in fact eliminates the $x$ variable.

In summary, among the C-equations we only need to consider the three-component equation $\mathrm{C}_{b_{0}}(2.12)$ for $b_{0} \neq 0$, and the two-component equations $\mathrm{C} 3_{b_{1}}(2.13 \mathrm{c} \mid 2.13 \mathrm{~d}$ ) (for arbitrary $\left.b_{1}\right)$. 


\subsection{Symmetries}

\subsection{1 $n \leftrightarrow m$ reflection symmetry}

As can be easily seen, all the equations are invariant under the $n \leftrightarrow m$ reflection, i.e., $\sim \leftrightarrow$, accompanied by $P \leftrightarrow Q$ parameter change.

\subsubsection{Reversal symmetry}

By reversal symmetry we mean symmetry under changing all tildes to undertildes and hats to underhats. More precisely, the indices change sign and then the generic point is renamed:

$$
x_{n+\nu, m+\mu} \mapsto x_{-n-\nu,-m-\mu}=x_{n^{\prime}-\nu, m^{\prime}-\mu},
$$

after which we can drop the primes. This reversal is then with respect to the lattice point $(n, m)$. In the notation where only shifts relative to $(n, m)$ are indicated ( $\operatorname{such}$ as $x_{0,1}$ ) we have $x_{\nu, \mu} \mapsto x_{-\nu,-\mu}$, after which we usually shift the whole equation.

B2: If we apply this reversal to B2 equation (2.3a) we have

$$
\widetilde{y}=x \widetilde{x}-z \stackrel{\text { reversal }}{\longmapsto} \underset{\sim}{y}=x \underset{\sim}{x}-z \stackrel{\text { shift }}{\longmapsto} y=\widetilde{x} x-\widetilde{z} .
$$

Thus we have reversal symmetry of (2.3a) (and (2.3b ) ) if we add the exchange $y \leftrightarrow z$. As for (2.3c), it turns out that we should also take $b_{0} \leftrightarrow-b_{0}$ and $(P, Q) \leftrightarrow(-P,-Q)$ which is natural for a reversal of direction. In summary, the B2 equations are reversal invariant if accompanied with

$$
\left(x, y, z, P, Q, b_{0}\right) \mapsto\left(x, z, y,-P,-Q,-b_{0}\right)
$$

C: Similarly for the C3 equations we have reversal symmetry if we include variable changes

$$
(x, y, z) \mapsto(-x, z, y) .
$$

For (2.5a, 2.5b) this is manifest, and also for most terms in (2.5c) but some terms need more computations. For example we have

$$
z \frac{P \widetilde{y} \widehat{z}-Q \widehat{y} \widetilde{z}}{\widetilde{z}-\widehat{z}} \mapsto z \frac{P \underset{\sim}{y} z-Q \underset{\wedge}{y} z}{z-\underline{\sim}} \mapsto \widehat{\widetilde{z}} \frac{P \widehat{y} \widetilde{z}-Q \widetilde{y} \widehat{z}}{\widehat{z}-\widetilde{z}} \mapsto \hat{\widetilde{y}} \frac{P \widehat{z} \widetilde{y}-Q \widetilde{z} \hat{y}}{\widehat{y}-\widetilde{y}},
$$

and to finish the computation we should still show that $z /(\widetilde{z}-\widehat{z})=\widehat{\widetilde{y}} /(\widehat{y}-\widetilde{y})$ but this follows by taking suitable linear combination of (2.5a), (2.5b) and their shifts.

The only other special term in C3 is $b_{0} x$ in (2.5c) which in this process changes to $-b_{0} \widehat{\widetilde{x}}$. However, by taking again a suitable combination of the (2.5a, 2.5b) and their shifts we can derive

$$
\widehat{\widetilde{x}}=x-z \frac{\widetilde{y} \widehat{z}-\widehat{z} \widetilde{y}}{\widetilde{z}-\widehat{z}},
$$

which combines with the $P, Q$ term. Thus if $b_{0} \neq 0$, we have reversal symmetry if we do the further parameter replacements

$$
\left(P, Q, b_{0}, b_{1}\right) \mapsto\left(P+b_{0}, Q+b_{0},-b_{0}\right) .
$$

The two-component equation $\mathrm{C} 33_{b_{1}}$ of $(2.13 \mathrm{c} \mid 2.13 \mathrm{~d})$ is also reversal symmetric. 
A2: The case of A2 is a bit more complicated: From (2.4a) we have

$$
\widetilde{y}=z \widetilde{x}-x \quad \mapsto \quad \underset{\sim}{y}=z \underset{\sim}{x}-x \quad \mapsto \quad y=\widetilde{z} x-\widetilde{x}
$$

but the usual map (2.21) does not take this to the original form. Dividing the last equation by $x \widetilde{x}$ yields $y /(x \widetilde{x})=\widetilde{z} / \widetilde{x}-1 / x$. Now it can be seen that we get the original form if instead of (2.21) we have

$$
(y, z, x) \mapsto(-z / x,-y / x, 1 / x) .
$$

For the $P, Q$ term we only need to show that $\widehat{\widetilde{x}} x /(\widehat{y} \widetilde{x}-\widetilde{y} \widehat{x})=1 /(\widehat{z}-\widetilde{z})$, which follows from (2.4a 2.4b) and their shifts. The form (2.23) suggest that the transformation can be simplified if we use another variable $w:=y / x$. Then we get the alternate form

A2-alt:

$$
\begin{aligned}
z-\widetilde{w} & =\frac{x}{\widetilde{x}}, \\
z-\widehat{w} & =\frac{x}{\widehat{x}}, \\
(\widetilde{z}-\widehat{z})(\widehat{\widetilde{z}}-w) & =P \frac{\widetilde{x}}{x}-Q \frac{\widehat{x}}{x} .
\end{aligned}
$$

These are reversal invariant with

$$
(w, z, x) \mapsto(-z,-w, 1 / x) .
$$

This is easy to show if one uses the formula

$$
\frac{\widehat{\widetilde{x}} x}{\widetilde{x} \widehat{x}}=\frac{\widetilde{w}-\widehat{w}}{\widetilde{z}-\widehat{z}}
$$

which follows from (2.24a, $2.24 \mathrm{~b})$.

\subsection{Initial values and evolution}

We will consider evolution starting from initial values given on a staircase, on which the inside corner points are given by $n+m=0$ and the outside corner points by $n+m=1$, see Figure 2 a). Another possible initial staircase is given by $n-m=0, n-m=-1$, see Figure 2 b). We have three sets of variables and several equations so we must look carefully which kind of initial values are necessary and make sense.

Let us first consider a staircase in the NW-SE direction, Figure 2 a). We assume that $x$ is given on all points of the staircase and to get started, also $z_{0,0}$. Then it is possible to compute, step by step, the red $y$ and $z$ values using the quasilinear equations, i.e., we can compute $y_{n, m}$ for $n+m=1$ and $z_{n, m}$ for $n+m=0, n \neq 0$. If the staircase is in the NE-SW direction, as in Figure 2 b), it is necessary to give $z_{n, m}$ for $n-m=0,-1, n \geq 0$ and $y_{n, m}$ for $n-m=0,-1, n \leq 0$.

After the above we still have three equations left for each square, by which we should be able to compute values at points where $n+m=2$ (red dots in Figure 2). But before that can be done for the staircase a) we need more initial values: In order to use the remaining quasilinear equations we also need $z_{n, m}, n+m=1$, and for the fully nonlinear equation, $y_{n, m}, n+m=0$. These additional necessary initial values are given in blue in Figure 2, For staircase b) all necessary initial values were needed already for filling in the staircase.

Since the systems are reversal symmetric the same initial values work for evolution in the opposite direction. 


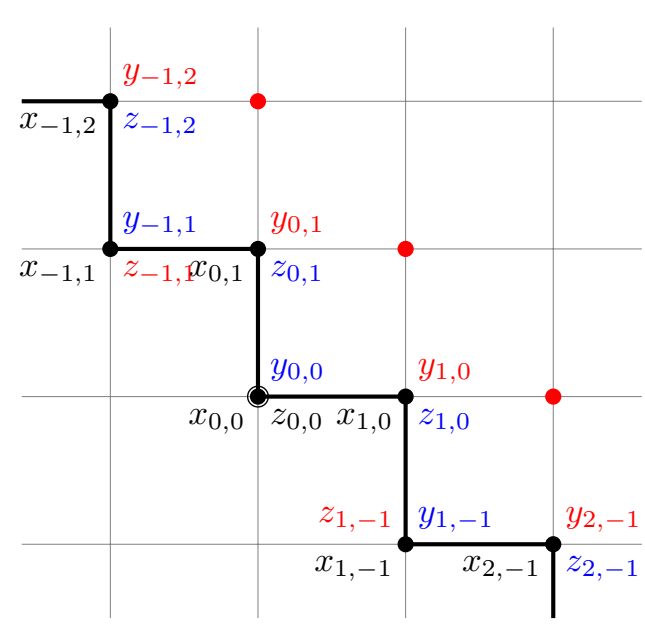

a)

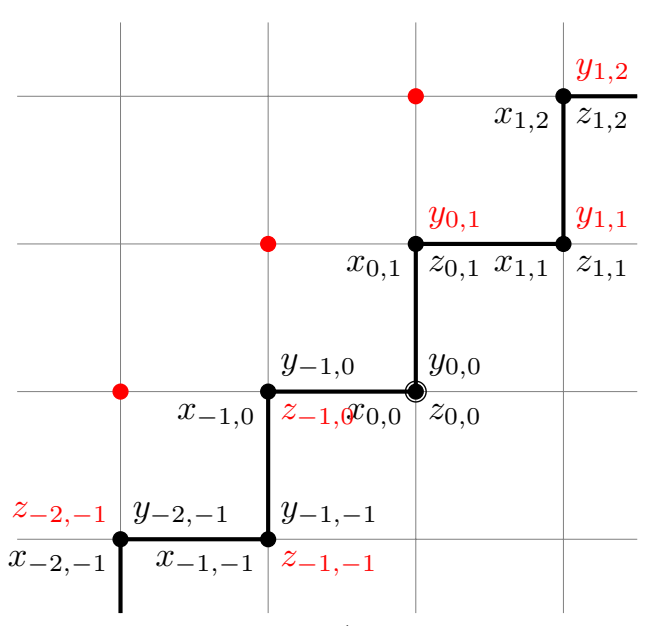

b)

Figure 2: The initial values given on the staircase. The black variables must be given in order to compute the red variables on the staircase using the quasilinear equations. In order to compute the values at red dots (one step in the evolution) it is also necessary to give the blue variables in case a).

\subsection{Connection with the direct-linearization results}

\subsubsection{The direct-linearization approach}

Many of the discrete Boussinesq equations discussed here were derived earlier (see [24, 27, 36, 37]) using the direct linearization scheme (DLA) of Capel, Nijhoff, Quispel et al. The results of Hietarinta [14] provided generalizations to the early results, but they were subsequently also derived from the DLA point of view in [41] by generalizing the dispersion relation.

The DLA was first proposed by Fokas and Ablowitz [8], and soon was developed to the study of discrete integrable systems [27, 28, 33]. In this approach, an infinite matrix is introduced via a linear integral equation with certain plane wave factors and discrete equations arise as closed forms of the shift relations of the elements of the matrix.

For the DBSQ equations, one first introduces an integral equation for infinite order column vector $\boldsymbol{u}(k)$ :

$$
\boldsymbol{u}(k)+\rho(k) \sum_{j=1}^{2} \int_{\Gamma_{j}} d \mu_{j}(l) \boldsymbol{u}(l) \sigma\left(-\omega_{j}(l)\right)=\rho(k) \boldsymbol{c}_{k}^{T},
$$

where $\Gamma_{j}$ and $d \mu_{j}(l)$ are contours and measures that need to be suitably chosen, $\boldsymbol{c}_{k}$ is an infinite order constant column vector $\left(\cdots, k^{-2}, k^{-1}, 1, k, k^{2}, \cdots\right)^{T}$, plane wave factors are

$$
\rho(k)=(p+k)^{n}(q+k)^{m} \rho^{(0)}(k), \quad \sigma\left(k^{\prime}\right)=\left(p-k^{\prime}\right)^{-n}\left(q-k^{\prime}\right)^{-m} \sigma^{(0)}\left(k^{\prime}\right),
$$

$\rho^{(0)}, \sigma^{(0)}$ are constants, $\omega_{j}(k)$ are defined through

$$
p^{3}+\alpha_{2} p^{2}-\left(k^{3}+\alpha_{2} k^{2}\right)=(p-k)\left(p-\omega_{1}(k)\right)\left(p-\omega_{2}(k)\right) .
$$

Then, introduce an $\infty \times \infty$ matrix $\boldsymbol{U}$ by

$$
\boldsymbol{U}=\sum_{j=1}^{2} \int_{\Gamma_{j}} d \mu_{j}(l) \sigma\left(-\omega_{j}(l)\right) \boldsymbol{u}(l) \boldsymbol{c}_{-\omega_{j}(l)}^{T} .
$$


After that one can define scalar functions

$$
S_{(a, b)}^{(i, j)}=\boldsymbol{e}^{T}(a+\Lambda)^{-1} \boldsymbol{U}(b-\Lambda)^{-1} \boldsymbol{e}, \quad i, j \in \mathbb{Z}, \quad a, b \in \mathbb{C},
$$

where $\boldsymbol{e}=(\cdots, 0,0,1,0,0, \cdots)^{T}$ in which only the center element is 1 , and $\Lambda=\left(\lambda_{i, j}\right)_{\infty \times \infty}$ in which $\lambda_{i, j}=\delta_{i, j-1}$. More explicitly we have

$$
\begin{array}{lll}
u^{(i, j)}=(-1)^{j} S_{(0,0)}^{(i, j)}, \quad s_{a, b}=S_{(a, b)}^{(-1,-1)}, & v_{a}=1-S_{(a, 0)}^{(-1,0)}, \quad w_{b}=1+S_{(0, b)}^{(0,-1)}, \\
s_{a}=a+S_{(a, 0)}^{(-1,1)}, \quad t_{b}=-b+S_{(0, b)}^{(1,-1)}, & r_{a}=a^{2}-S_{(a, 0)}^{(-1,2)}, \quad z_{b}=b^{2}+S_{(0, b)}^{(2,-1)} .
\end{array}
$$

DBSQ equations arise from closed forms of the shift relations of the above elements (cf. [41]).

Due to the different origin, the equations from DLA appear in a different gauge and in this section we elaborate the connections.

\subsubsection{B2}

Let us focus on B2 equation (2.3), in which we may assume that $b_{1}=0$, as mentioned before. If we use on (2.3) the transformation [41]

$$
\begin{aligned}
& x=u^{(0,0)}-x_{0}, \\
& y=u^{(0,1)}-x_{0} u^{(0,0)}+y_{0}, \\
& z=u^{(1,0)}-x_{0} u^{(0,0)}+z_{0},
\end{aligned}
$$

where

$$
\begin{aligned}
& x_{0}=n p+m q+c_{1}, \\
& y_{0}=\frac{1}{2}\left(n p+m q+c_{1}\right)^{2}-\frac{1}{2}\left(n p^{2}+m q^{2}+c_{2}\right)-c_{3}, \\
& z_{0}=\frac{1}{2}\left(n p+m q+c_{1}\right)^{2}+\frac{1}{2}\left(n p^{2}+m q^{2}+c_{2}\right)+c_{3}
\end{aligned}
$$

and $c_{j}(j=1,2,3)$ are constants, we obtain

$$
\begin{aligned}
p \widetilde{u}^{(0,0)}-\widetilde{u}^{(0,1)}=p u^{(0,0)}+u^{(1,0)}-\widetilde{u}^{(0,0)} u^{(0,0)} & \\
q \widehat{u}^{(0,0)}-\widehat{u}^{(0,1)}=q u^{(0,0)}+ & u^{(1,0)}-\widehat{u}^{(0,0)} u^{(0,0)}, \\
-\frac{P-Q}{p-q+\widehat{u}^{(0,0)}-\widetilde{u}^{(0,0)}} & =\frac{G_{3}(-p,-q)}{q-p}+\left(p+q+b_{0}\right)\left(u^{(0,0)}-\widehat{\widetilde{u}}^{(0,0)}\right) \\
& -u^{(0,0)} \widehat{\widetilde{u}}^{(0,0)}+\widehat{\widetilde{u}}^{(1,0)}+u^{(0,1)},
\end{aligned}
$$

where

$$
\begin{aligned}
& G_{3}(a, b)=g_{3}(a)-g_{3}(b), \\
& g_{3}(a)=a^{3}+\alpha_{2} a^{2},
\end{aligned}
$$

and $b_{0}=-\alpha_{2}$. This agrees with the DLA result (Eqs.(32a,33) in Ref. [41]) provided that we parameterise $P, Q$ in terms of $p, q$ as follows:

$$
P=g_{3}(-p), \quad Q=g_{3}(-q),
$$


with $b_{0}=-\alpha_{2}$. Note that one can always replace $-p$ and $-q$ by $a$ and $b$, respectively, and then get the parametrisation used in [19] for getting soliton solutions.

The above parametrization of $P, Q$ provides also a connection to the lattice potential $\mathrm{KdV}$ equation [26,28]: Using (2.30, 2.29b) and then taking the singular limit $b_{0} \rightarrow \infty$ we get from (2.3c)

$$
(x-\widehat{\widetilde{x}})(\widetilde{x}-\widehat{x})=-p^{2}+q^{2} .
$$

This one-component equation appears as H1 equation in the ABS list [1].

Here is a second alternative form of $(2.3)$ in the special case $b_{0}=0$ :

$$
\begin{aligned}
& \omega\left(\widehat{u}^{(0,1)}-\widetilde{u}^{(0,1)}\right)=p \widetilde{u}^{(0,0)}-q \widehat{u}^{(0,0)}-u^{(0,0)}\left(p-q+\widehat{u}^{(0,0)}-\widetilde{u}^{(0,0)}\right),\left(\omega=e^{\frac{2 \pi i}{3}}\right), \\
& \widehat{u}^{(1,0)}-\widetilde{u}^{(1,0)}=q \widetilde{u}^{(0,0)}-p \widehat{u}^{(0,0)}+\widehat{\widetilde{u}}^{(0,0)}\left(p-q+\widehat{u}^{(0,0)}-\widetilde{u}^{(0,0)}\right), \\
& \widehat{\widetilde{u}}^{(1,0)}-\omega u^{(0,1)}=p q-\left(p+q+u^{(0,0)}\right)\left(p+q-\widehat{\widetilde{u}}^{(0,0)}\right)+\frac{p^{3}-q^{3}}{p-q+\widehat{u}^{(0,0)}-\widetilde{u}^{(0,0)}} .
\end{aligned}
$$

This is Eq.(5.3.12) in Ref. [37] as well as Eqs.(2.15a-c) with $N=3$ in Ref. [27]. In the above system, (2.32a and $(2.32 \mathrm{~b})$ can be obtained through $(2.28 \mathrm{a})-(2.28 \mathrm{~b})$ and $(2.28 \mathrm{a})-(2.28 \mathrm{~b})$, and (2.32c $)$ is $(2.28 \mathrm{c})$ with $b_{0}=0$, in addition, $u^{(0,1)} \rightarrow-\omega u^{(0,1)}$.

Note that the alternative forms (2.28) with (2.29) and (2.32) have the "background solution" $u^{(k, l)}=0$, corresponding to (2.27) for (2.3). Thus these alternative forms will be useful once we start to construct soliton solutions in Section 6 and continuum limits in Section 4 .

\subsubsection{A2}

For the A2 equation (2.4) (without the removable parameter $b_{0}$ (cf. (2.8) )) several alternative forms have been presented in the literature.

The form

$$
\begin{aligned}
& \widetilde{s}_{a}=\left(p+u^{(0,0)}\right) \widetilde{v}_{a}-(p-a) v_{a} \\
& \widehat{s}_{a}=\left(q+u^{(0,0)}\right) \widehat{v}_{a}-(q-a) v_{a}, \\
& \left(p+q-\widehat{\widetilde{u}}^{(0,0)}+\frac{s_{a}}{v_{a}}-\alpha_{2}\right)\left(p-q+\widehat{u}^{(0,0)}-\widetilde{u}^{(0,0)}\right)=p_{a} \frac{\widetilde{v}_{a}}{v_{a}}-q_{a} \frac{\widehat{v}_{a}}{v_{a}}
\end{aligned}
$$

was derived from direct linearisation approach (see Eq.(30) in Ref. [41]), here $p_{a}$ and $q_{a}$ are defined as

$$
p_{a}=\frac{-G_{3}(-p,-a)}{p-a}, \quad q_{a}=\frac{-G_{3}(-q,-a)}{p-a} .
$$

The transformation between Eq.(2.4) and Eq.(2.33) is given by

$$
v_{a}=\frac{x}{x_{a}}, \quad u^{(0,0)}=z-z_{0}, \quad s_{a}=\frac{1}{x_{a}}\left(y-v_{a} y_{a}\right),
$$

where

$$
\begin{aligned}
x_{a} & =(p-a)^{-n}(q-a)^{-m} c_{1}, \\
z_{0} & =\left(c_{3}-p\right) n+\left(c_{3}-q\right) m+c_{2}, \\
y_{a} & =x_{a}\left(z_{0}-c_{3}\right),
\end{aligned}
$$


and where $c_{1}, c_{2}$ are constants, $c_{3}=\alpha_{2} / 3$ (if $\left.b_{0}=0\right)$ and

$$
P=-G_{3}(-p,-a), \quad Q=-G_{3}(-q,-a) .
$$

Note that the above $P, Q$ can be equivalently reparameterised as

$$
P=G_{3}(p, a), Q=G_{3}(q, a)
$$

if we take $c_{3}=-\alpha_{2} / 3$ in (2.36). Either convention can be adopted.

Another system related to A2 was given in Eqs.(4.22a, 4.21b) of Ref. [24], i.e.

$$
\begin{aligned}
& \widetilde{s} \widetilde{v}=(p+u) \widetilde{v}-p v \\
& \widehat{s} \widehat{v}=(p+u) \widehat{v}-p v \\
& (p+q+s-\widehat{\widetilde{u}})(p-q+\widehat{u}-\widetilde{u})=\frac{p^{2} \widetilde{v}-q^{2} \widehat{v}}{v}
\end{aligned}
$$

which can be derived from (2.33) by taking

$$
s_{a}=s v, u_{0}=u, v_{a}=v, a=0, \alpha_{2}=0 .
$$

Then there is the system

$$
\begin{aligned}
& p-q+\widehat{u}-\widetilde{u}=\frac{(p-a) \widehat{v}-(q-a) \widetilde{v}}{\widehat{\widetilde{v}}} \\
& p-q+\widehat{s}-\widetilde{s}=(p-a) \frac{v}{\widetilde{v}}-(q-a) \frac{v}{\widehat{v}} \\
& (p+q+s-\widehat{\widetilde{u}})(p-q+\widehat{u}-\widetilde{u})=\frac{p_{a} \widetilde{v}-q_{a} \widehat{v}}{v}
\end{aligned}
$$

which is given by Eqs.(A.4a,b,c) in [27], and named as the "Toda-MBSQ equation". In fact, (2.41a) and (2.41b) can be derived from (2.33a) and (2.33b), by using

$$
s_{a}=s v, v_{a}=v, u^{(0,0)}=u, \alpha_{2}=0,
$$

and then eliminating $s$ and $u$, respectively.

Finally, eliminating $s_{a}$ from (2.33a $)$ and (2.33b) yields

$$
p-q+\widehat{u}_{0}-\widetilde{u}_{0}=\frac{(p-a) \widehat{v}_{a}-(q-a) \widetilde{v}_{a}}{\widehat{\widetilde{v}}_{a}}
$$

which, together with (2.33a ) and (2.33c) with $\alpha_{2}=0$, gives the system $\{(5.3 .7 \mathrm{a}),(5.3 .14)$, (5.3.15)\} in Ref. [37].

\subsubsection{C3}

The C3 equation is related to the following equation derived by DLA [41]

$$
\begin{aligned}
& (p-a) S_{a, b}-(p-b) \widetilde{S}_{a, b}=\widetilde{v}_{a} w_{b}, \\
& (q-a) S_{a, b}-(q-b) \widehat{S}_{a, b}=\widehat{v}_{a} w_{b}, \\
& v_{a} \widehat{\widetilde{w}}_{b}=w_{b} \frac{\frac{p_{a}}{p-b} \widehat{w}_{b} \widetilde{v}_{a}-\frac{q_{a}}{q-b} \widetilde{w}_{b} \widehat{v}_{a}}{(p-b) \widetilde{w}_{b}-(q-b) \widehat{w}_{b}}+\frac{G_{3}(-a,-b)}{(p-b)(q-b)} S_{a, b},
\end{aligned}
$$


$S_{a, b}=s_{a, b}-1 /(a-b)$ and the connection between the two equations is [4]

$$
\begin{aligned}
& S_{a, b}=\left(\frac{p-a}{p-b}\right)^{n}\left(\frac{q-a}{q-b}\right)^{m} x, \\
& v_{a}=-(p-a)^{n}(q-a)^{m} y \\
& w_{b}=(p-b)^{-n}(q-b)^{-m} z
\end{aligned}
$$

where

$$
P=-G_{3}(-p,-a), \quad Q=-G_{3}(-q,-b), \quad b_{0}=G_{3}(-a,-b),
$$

and $G_{3}(a, b), p_{a}$ and $q_{a}$ are defined as before.

Note that since $\alpha_{2}$ is arbitrary we can replace $\alpha_{2}$ by $-\alpha_{2}$ and thus in C3 (2.12) $P, Q$ and $b_{0}$ can be reparameterised as

$$
P=G_{3}(p, a), \quad Q=G_{3}(q, b), \quad b_{0}=-G_{3}(a, b),
$$

where $G_{3}(a, b)$ is defined as (2.29).

\section{Two- and one-component forms}

So far we have discussed the BSQ equations in their three-component forms, e.g., in terms of $x, y, z$, or $u^{(0,0)}, u^{(1,0)}, u^{(0,1)}$ etc. As explained in Section 2.5, Figure 2, for the discrete BSQ equations it is then necessary to give the initial values for two components on an staircase-like configuration before the next step in the evolution can be computed. An alternative formulation of second order time evolution is to use only one component and give initial values on two consecutive staircases as in Figure 3, In that case the equation usually involves the points within a $3 \times 3$ stencil. (Note that in the two-component case it is not necessary that the equations are defined on a square, only that the next step in the evolution can be calculated once values on the staircase are given.)

In this section we will derive two- and one-component forms of these equations. The process of variable elimination can also be interpreted as a Bäcklund transformation (BT). Assuming we have the following situation:

$$
G[x]=0 \stackrel{\text { eliminate } y}{\longleftarrow}\left\{\begin{array}{l}
A[x, y]=0 \\
B[x, y]=0
\end{array} \stackrel{\text { eliminate } x}{\longrightarrow} H[y]=0\right.
$$

Then we say that the pair $\{A[x, y]=0, B[x, y]=0\}$ provides a BT between $G[x]=0$ and $H[y]=0$. Another way of looking at the above is to consider the pair $\{A[x, y]=$ $0, B[x, y]=0\}$ as two equations for one variable $x$, which can be solved, provided that the other variable $y$ satisfies some "integrability condition". In the context of PDE's this is a familiar situation. For example, from the pair $\partial_{x} \psi=A(x, y), \partial_{y} \psi=B(x, y)$ we can solve $\psi$ only if $\partial_{x} B=\partial_{y} A$. In more general cases this problem of "formal integrability" or "involutivity" of a set of PDE's can become quite complicated 1 and the problem has

\footnotetext{
${ }^{1}$ The idea is to compute differential consequences of the initial equations and try to find an "involutive completion" after which the new differential consequences are just prolongations and produce no genuine new equations. One of the difficult problems is to decide when one can stop this process (this is apparently based on Spencer cohomology).
} 


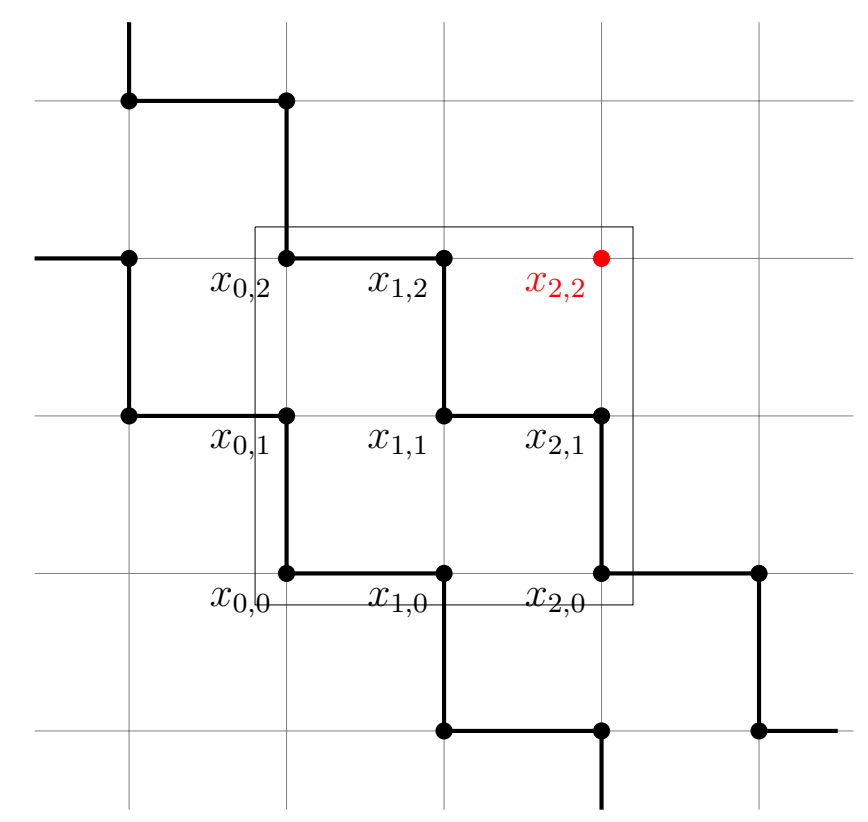

Figure 3: For one-component second order time evolution initial values can be given on two consecutive staircases. The evolution equation will then be given on the indicated 9 -point stencil.

been analysed at length in the mathematics literature (the reader is referred to a recent monograph [35]).

Now we have a partial difference version of the same thing: we can integrate one of the variables, say $x$ if the other variable, say $y$, satisfies some $\mathrm{P} \Delta \mathrm{E}$ (condition for integrability), which we should find. To do that we may need several shifted consequences of the original equations, which provide new equations but also new variables. The hope is that we eventually have a sufficient number of equations to solve for all shifts of $x$ and still have one more equation that gives a condition on the other variable 2

Since we will need several shifts in both directions we will use the notation where we give as subscripts just the shifts with respect to the basic position $(n, m)$, for example $\widetilde{y}=y_{1,0}$.

\subsection{Generalities about the elimination process}

Before studying the specific equations we can make some general observations. Our starting point is the set of three equations for three variables, A2, B2, or C3. Since we always have an equation with un-shifted $z$ it is easy to solve for that variable and use it in the remaining ones. Similarly for $y$, we just need to apply shifts, although due to reversal symmetry we do not have to consider this case. Thus we can easily construct a two component pair of equations in $y$ and $x$ or in $z$ and $x$.

The situation with $x$ is different, because the first two equations contain $x_{0,0}, x_{1,0}$, $x_{0,1}$. We can use these to eliminate all shifted $x$ and the resulting equation would then be a polynomial in $x_{0,0}$. In the optimal case the $x_{0,0}$-dependent part would factor out, but this does not always happen. Furthermore it turns out that sometimes it is beneficial to

\footnotetext{
${ }^{2}$ After this it remains to prove that all the remaining equations are satisfied due to this one condition (although this is never done in practice).
} 


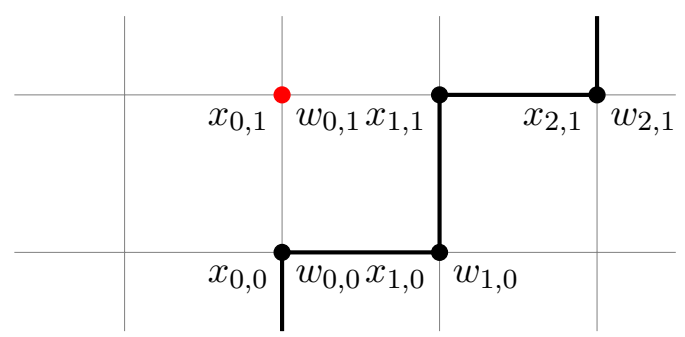

Figure 4: Equations (3.2a $)$ and (3.2c) allow evolution in the NW direction, even if initial values outside the square are needed.

absorb some $x$-dependence into $y$ by writing the equation in terms of $w:=y / x$.

As discussed in Section 2.4.2, all of our equations are reversal symmetric, which in particular exchanges $z$ and $y$. We will therefore only need to construct two-component forms in terms of $(y, z)$ and $(y, x)$ or $(z, x)$.

\section{$3.2 \quad \mathrm{~A} 2$}

We take the A2 equation in the alternate form (2.24) with $w:=y / x$ :

$$
\begin{aligned}
z_{0,0}-w_{1,0} & =\frac{x_{0,0}}{x_{1,0}}, \\
z_{0,0}-w_{0,1} & =\frac{x_{0,0}}{x_{0,1}} \\
\left(z_{1,0}-z_{0,1}\right)\left(z_{1,1}-w_{0,0}\right) & =P \frac{x_{1,0}}{x_{0,0}}-Q \frac{x_{0,1}}{x_{0,0}},
\end{aligned}
$$

because it is reversal symmetric, and because in this form $x$ appears homogeneously and is therefore easier to eliminate.

A2 in terms of $x$ and $w:=y / x$ : Solving for $z_{0,0}$ from (3.1a) we get from (3.1b) and (3.1c)

$$
\begin{aligned}
& w_{1,0}-w_{0,1}=\frac{x_{0,0}}{x_{0,1}}-\frac{x_{0,0}}{x_{1,0}} \\
& w_{2,1}-w_{0,0}=\frac{x_{1,1}}{x_{0,0}} \frac{P x_{1,0}-Q x_{0,1}}{x_{1,0}-x_{0,1}}-\frac{x_{1,1}}{x_{2,1}} .
\end{aligned}
$$

This is suitable for next eliminating $w$, but if we want to eliminate $x$ then it is best to first eliminate $x_{0,0}$ from (3.2b) using (3.2a) which gives the alternative form

$$
\left(w_{2,1}-w_{0,0}+\frac{x_{1,1}}{x_{2,1}}\right)\left(w_{1,0}-w_{0,1}\right)=P \frac{x_{1,1}}{x_{0,1}}-Q \frac{x_{1,1}}{x_{1,0}} .
$$

The corresponding equation for $z, x$ can be obtained by reversal symmetry, together with (2.25). Note that equations (3.2b) and (3.2c) are not defined on the basic square but contain an extra point at $(2,1)$. Nevertheless they allow evolution from a staircase initial conditions, as shown in Figure 4. 
A2 in terms of $z$ and $w:=y / x$ : In order to eliminate $x$-dependence from (3.1a) and (3.1b) we need to take shifts and ratios, leading to [9, 31]

$$
\begin{aligned}
\frac{w_{1,0}-z_{0,0}}{w_{0,1}-z_{0,0}} & =\frac{w_{1,1}-z_{0,1}}{w_{1,1}-z_{1,0}}, \\
\left(w_{0,0}-z_{1,1}\right)\left(z_{1,0}-z_{0,1}\right) & =\frac{P}{w_{1,0}-z_{0,0}}-\frac{Q}{w_{0,1}-z_{0,0}} .
\end{aligned}
$$

The second equation has the alternative form obtained by eliminating the $z_{0,0}$-dependency

$$
\left(w_{0,0}-z_{1,1}\right)\left(w_{1,0}-w_{0,1}\right)=\frac{P}{w_{1,1}-z_{0,1}}-\frac{Q}{w_{1,1}-z_{1,0}} .
$$

Equations (3.3b) and (3.3c) are connected by

$$
(P, Q, n, m, w, z) \rightarrow(-P,-Q,-n,-m, z, w) .
$$

The equations (3.3) are defined on the basic quadrilateral and are 3-dimensionally consistent; the triply shifted quantities are given in the Appendix.

A2 in terms of $x$ only from (3.2a ) and (3.2b): From equations (3.2a $),(3.2 \mathrm{~b})$ it is easy to derive an equation for $x$ alone. These equations are of the following type

$$
w_{1,0}-w_{0,1}=A_{0,0}, \quad w_{2,1}-w_{0,0}=B_{0,0},
$$

and by taking suitable shifts one can eliminate $w$ and derive the "integrability condition"

$$
B_{1,0}-B_{0,1}=A_{2,1}-A_{0,0}
$$

When this is calculated we get an equation on a $3 \times 3$ stencil (cf. Figure 3) (see Eq.(A.5) in [27], (5.2) in [23], Eq.(4.9) in [24] and Eq.(5.7.6) in [37]).

$$
\left(\frac{P x_{1,1}-Q x_{0,2}}{x_{0,2}-x_{1,1}}\right) \frac{x_{1,2}}{x_{0,1}}-\left(\frac{P x_{2,0}-Q x_{1,1}}{x_{1,1}-x_{2,0}}\right) \frac{x_{2,1}}{x_{1,0}}=\frac{x_{0,0}}{x_{1,0}}-\frac{x_{0,0}}{x_{0,1}}-\frac{x_{1,2}}{x_{2,2}}+\frac{x_{2,1}}{x_{2,2}} .
$$

This equation is reversal symmetric with (2.25) and only changes sign.

A2 in terms of $w$ only from (3.3a) and (3.3c): In order to derive other one component equations one needs a slightly more complicated sequence of elimination steps. In order to eliminate $z$ from (3.3a) and (3.3c) we observe that the $z_{n, m}$ that appear in these equations are located on the lattice as given in Figure 5.1, where (a) corresponds to (3.3c) and (b) to (3.3a).

The elimination process then goes on as follows: Assume that the $z$-values at points 1 and 2 of Figure 5.2 are arbitrary (say, $z_{0,0}$ and $z_{1,0}$ ), then using (b) we can compute the value at point 3 ; we denote this process by $(1 ; 2) \stackrel{(b)}{\longrightarrow} 3$. There are two routes to compute the $z$-value at point 5 :

$$
\begin{array}{lll}
(1 ; 2) \stackrel{(b)}{\longrightarrow} 3, & (2 ; 3) \stackrel{(a)}{\longrightarrow} 4, & (4 ; 2) \stackrel{(b)}{\longrightarrow} 5, \\
(1 ; 2) \stackrel{(a)}{\longrightarrow} 6, & (2 ; 6) \stackrel{(b)}{\longrightarrow} 7, \quad(2 ; 7) \stackrel{(a)}{\longrightarrow} 5
\end{array}
$$




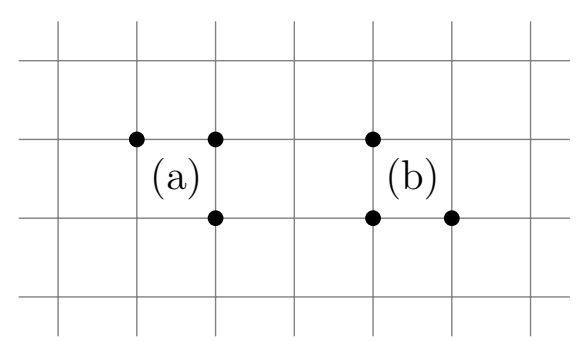

Figure 51

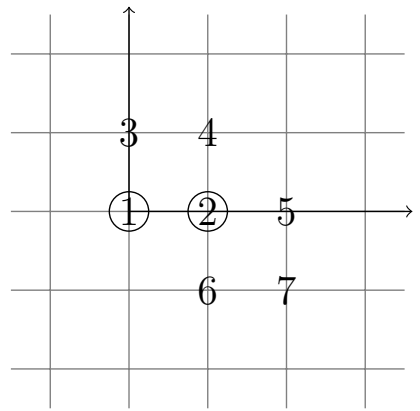

Figure 5.2

Figure 5: If equations (a) and (b) have variables located in the lattice as in Figure 5,1 then in the elimination process one needs to consider 7 points as given in Figure 5.2.

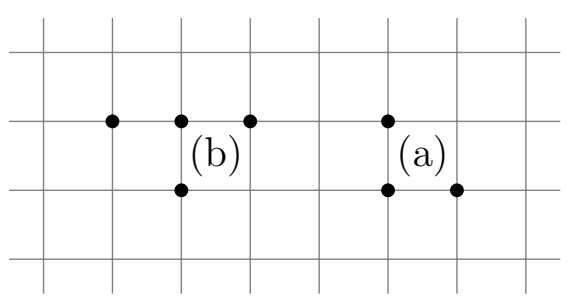

Figure 6, 1

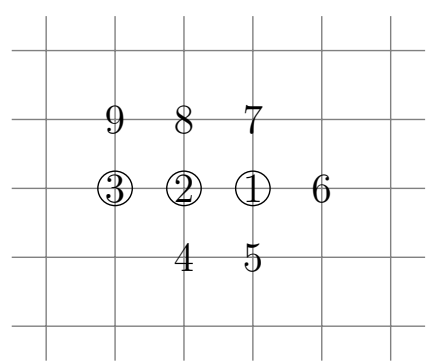

Figure 6 , 2

Figure 6: If equations (a) and (b) have variables located in the lattice as in Figure 6, 1 then in the elimination process one need to consider 9 points as given in Figure 6. 2.

The resulting two values for $z$ at 5 must be the same, for the arbitrary values of $z$ at points 1 and 2. Equating the two computed values yields a rational expression in the arbitrary initial values $z_{0,0}, z_{1,0}$. In this case the numerator does not contain the initial values and gives the equation. The necessary polynomial algebra is straightforward but tedious and is best done using a computer algebra system (such as REDUCE [13] or Mathematica). The result is (see (1.3) in [27], (5.3) in [23], (4.18) in [24] and Eq. (5.7.3) in [37].)

$$
\frac{P-Q}{w_{2,0}-w_{1,1}}-\frac{P-Q}{w_{1,1}-w_{0,2}}-\left(w_{2,2}-w_{0,1}\right)\left(w_{2,1}-w_{1,2}\right)-\left(w_{0,0}-w_{2,1}\right)\left(w_{1,0}-w_{0,1}\right)=0 .
$$

The equation for $z$ alone can be obtained by reversal symmetry and its form is identical to (3.6) except for sign changes (cf.(2.25) ).

A2 in terms of $w$ only from (3.2a) and (3.2c): We could also eliminate $x$ from (3.2) in order to obtain an equation in $w$. In the present A2 case it is not necessary because we did already derive the $w$ equation using another sequence of eliminations. However, in some later cases we need this different kind of elimination process, so we will do it here as an exercise with guaranteed success.

First note that the $x$ variables appear in lattice positions as illustrated Figure 6, with Figure 6.1(a) corresponding to (3.2a) and Figure 6.1(b) to (3.2c). The elimination 
process is now as follows: We assume that $x$ values at circled points 1,2,3 are given and then compute the other $x$ values as follows

$$
\begin{aligned}
(1 ; 2 ; 3) \stackrel{(b)}{\longrightarrow} 4, \quad(2 ; 4) \stackrel{(a)}{\longrightarrow} 5, \quad(1 ; 2 ; 5) \stackrel{(b)}{\longrightarrow} 6, \quad(6 ; 1) \stackrel{(a)}{\longrightarrow} 7, \\
(1,2) \stackrel{(a)}{\longrightarrow} 8, \quad(2 ; 3) \stackrel{(a)}{\longrightarrow} 9, \quad(2 ; 8 ; 9) \stackrel{(b)}{\longrightarrow} 7 .
\end{aligned}
$$

The two values of $x$ at point 7 must be the same, which gives us an equation which should hold for arbitrary values of $x$ at 1,2,3. When the computations are done the result is (3.6), as expected.

\section{$3.3 \quad$ B2}

The B2 equation is given by

$$
\begin{aligned}
& y_{1,0}+z_{0,0}=x_{0,0} x_{1,0}, \\
& y_{0,1}+z_{0,0}=x_{0,0} x_{0,1}, \\
& y_{0,0}+z_{1,1}=x_{0,0} x_{1,1}+b_{0}\left(x_{1,1}-x_{0,0}\right)+\frac{P-Q}{x_{1,0}-x_{0,1}} .
\end{aligned}
$$

Note that equations $(3.7 \mathrm{a} / 3.7 \mathrm{~b})$ are similar to $(3.1 \mathrm{a}, 3.1 \mathrm{~b})$ and therefore the derivation of two-component forms is similar.

B2 in terms of $y$ and $x$ : Solving $z_{0,0}$ from (3.7a and using in the other equations yields

$$
\begin{aligned}
& y_{1,0}-y_{0,1}=x_{0,0}\left(x_{1,0}-x_{0,1}\right), \\
& y_{0,0}-y_{2,1}=\left(x_{0,0}-x_{2,1}\right) x_{1,1}+b_{0}\left(x_{1,1}-x_{0,0}\right)+\frac{P-Q}{x_{1,0}-x_{0,1}} .
\end{aligned}
$$

Another two-component form is obtained if we replace $y$ by $w:=y+z$ and after that eliminate $z$. The result is

$$
\begin{aligned}
w_{0,1}-w_{1,0}+\left(x_{0,0}+x_{1,1}\right)\left(x_{0,1}-x_{1,0}\right) & =0, \\
\frac{P-Q-x_{1,0} w_{1,0}+x_{0,1} w_{0,1}}{x_{1,0}-x_{0,1}}-w_{0,0}-w_{1,1}+b_{0}\left(x_{1,1}-x_{0,0}\right) & \\
+\left(x_{0,0}+x_{1,1}\right)\left(x_{1,0}+x_{0,1}\right)+x_{0,0} x_{1,1} & =0 .
\end{aligned}
$$

A notable difference with (3.8) is that this is defined on an elementary quadrilateral; it has the CAC property. This form was presented in [34 but its relation to B2 was left open.

B2 in terms of $x$ only from (3.8a) and (3.8b): The structure of equations (3.8a $)$ and $(3.8 \mathrm{~b})$ is as in (3.4a $)$ so that we get the one-component equation using (3.4b):

$$
\begin{aligned}
(P-Q) & \left(\frac{1}{x_{2,0}-x_{1,1}}-\frac{1}{x_{1,1}-x_{0,2}}\right)+b_{0}\left(x_{0,1}-x_{1,0}+x_{2,1}-x_{1,2}\right) \\
& -\left(x_{2,2}-x_{0,1}\right)\left(x_{2,1}-x_{1,2}\right)-\left(x_{0,0}-x_{2,1}\right)\left(x_{1,0}-x_{0,1}\right)=0 .
\end{aligned}
$$

This equation is a generalization ( $b_{0}$-terms) of (3.6) derived for A2.

Attempts to eliminate $x$ seem to lead into very complicated equations. 


\section{$3.4 \quad \mathrm{C} 3$}

As noted in Section 2.3 we only need to consider C3 equation

$$
\begin{aligned}
& y_{1,0} z_{0,0}=x_{1,0}-x_{0,0} \\
& y_{0,1} z_{0,0}=x_{0,1}-x_{0,0}, \\
& z_{1,1} y_{0,0}=b_{0} x_{0,0}+b_{1}+z_{0,0} \frac{P y_{1,0} z_{0,1}-Q y_{0,1} z_{1,0}}{z_{1,0}-z_{0,1}}
\end{aligned}
$$

for the three-component case $b_{0} \neq 0, b_{1}=0$ and for the two component case $b_{0}=0, b_{1}$ arbitrary. We can treat them together for some computations.

C3 in terms of $x, y$ : After solving for $z$ from (3.11a) and using it in (3.11b) and (3.11c) we get the two-component form

$$
\begin{aligned}
y_{1,0}\left(x_{0,1}-x_{0,0}\right) & =y_{0,1}\left(x_{1,0}-x_{0,0}\right) \\
y_{0,0}\left(x_{2,1}-x_{1,1}\right) & =y_{2,1}\left(b_{0} x_{0,0}+b_{1}\right) \\
& +y_{2,1} \frac{\left(x_{1,1}-x_{0,1}\right)\left(x_{0,0}-x_{1,0}\right) P-\left(x_{1,1}-x_{1,0}\right)\left(x_{0,0}-x_{0,1}\right) Q}{x_{1,0}-x_{0,1}} .
\end{aligned}
$$

This form is well suited for eliminating $y$ next, because it is linear in $y$, but if we would like to eliminate $x$ the following alternative would be useful:

$$
\left(x_{2,1}-x_{1,1}\right) \frac{y_{0,0}}{y_{2,1}}=\left(b_{0} x_{0,0}+b_{1}\right)-\frac{\left(x_{1,1}-x_{0,1}\right) y_{1,0} P-\left(x_{1,1}-x_{1,0}\right) y_{0,1} Q}{y_{1,0}-y_{0,1}},
$$

because it is linear in $x$. These equations are defined on a configuration given in Figure 4 $(w \leftrightarrow y)$. The equations for $x, z$ are the same as above, and can be obtained by reversal, cf.(2.212.22).

C3 in terms of $x$ only: Equations (3.12a, 3.12b) have the form

$$
\frac{y_{1,0}}{y_{0,1}}=A_{0,0}, \quad \frac{y_{0,0}}{y_{2,1}}=B_{0,0}
$$

The dependence is similar to (3.4), except that instead of an additive case we now have a multiplicative case. The integrability condition is

$$
\frac{A_{2,1}}{A_{0,0}}=\frac{B_{0,1}}{B_{1,0}}
$$

which yields

$$
\begin{aligned}
& \frac{\left(x_{2,2}-x_{1,2}\right)\left(x_{0,2}-x_{1,1}\right)\left(x_{0,1}-x_{0,0}\right)}{\left(x_{2,2}-x_{2,1}\right)\left(x_{1,1}-x_{2,0}\right)\left(x_{1,0}-x_{0,0}\right)}= \\
& \frac{\left(x_{1,1}-x_{0,2}\right)\left(b_{0} x_{0,1}+b_{1}\right)+\left(x_{1,2}-x_{0,2}\right)\left(x_{0,1}-x_{1,1}\right) P-\left(x_{1,2}-x_{1,1}\right)\left(x_{0,1}-x_{0,2}\right) Q}{\left(x_{2,0}-x_{1,1}\right)\left(b_{0} x_{1,0}+b_{1}\right)+\left(x_{2,1}-x_{1,1}\right)\left(x_{1,0}-x_{2,0}\right) P-\left(x_{2,1}-x_{2,0}\right)\left(x_{1,0}-x_{1,1}\right) Q} .
\end{aligned}
$$

Note that this is invariant under $n \leftrightarrow m, P \leftrightarrow Q$, and reversal symmetric with (2.22). 
C3 in terms of $y$ only: The dependence on $x$ in (3.12a) and (3.12c) is linear and of the form given in Figure 6, and using that method we can eliminate $x$. The resulting equation in terms of $y$ is the same as (3.5) in terms of $x$. Note in particular that dependence on the parameters $b_{0}, b_{1}$ drops out from the $y$-equation, while it remains in the $x$-equation.

C3 in terms of $y$ and $z$ : The method of eliminating $x$ depends sensitively on the additional $x$-dependent term. We need to consider separately the two cases.

- $b_{1}$ arbitrary, $b_{0}=0$. In this case equation (3.11c) does not depend on $x$ at all, while form (3.11a) and (3.11b) $x$ can be easily eliminated, leaving

$$
\begin{aligned}
y_{1,1}\left(z_{1,0}-z_{0,1}\right) & +z_{0,0}\left(y_{1,0}-y_{0,1}\right)=0, \\
y_{0,0} z_{1,1} & =b_{1}+z_{0,0} \frac{P y_{1,0} z_{0,1}-Q y_{0,1} z_{1,0}}{z_{1,0}-z_{0,1}} .
\end{aligned}
$$

Note that by the gauge transformation

$$
z=w p^{n} q^{m}, \quad y=-v p^{-n} q^{-m},
$$

with $P=p^{3}, Q=q^{3}$ and $b_{1}=b_{1}^{\prime} p q$, we will get from (3.14)

$$
\frac{p \widetilde{w}-q \widehat{w}}{w}=\frac{p \widehat{v}-q \widetilde{v}}{\widehat{\widetilde{v}}}=\frac{p \widetilde{v} \widehat{w}-q \widehat{v} \widetilde{w}}{b_{1}^{\prime}+v \widehat{\widetilde{w}}} .
$$

When $b_{1}^{\prime}=0$ we get

$$
\frac{p \widetilde{w}-q \widehat{w}}{w}=\frac{p \widehat{v}-q \widetilde{v}}{\widehat{\widetilde{v}}}=\frac{p \widetilde{v} \widehat{w}-q \widehat{v} \widetilde{w}}{v \widehat{\widetilde{w}}}
$$

which was already presented in [24].

- $b_{1}=0, b_{0} \neq 0$. In this case it is best to absorb some $x$ into $y$ by defining $w:=y / x$. Then (3.11a) and (3.11b) yield

$$
w_{1,1} z_{0,0}\left(w_{1,0} z_{1,0}-w_{0,1} z_{0,1}\right)-w_{1,1}\left(z_{1,0}-z_{0,1}\right)-z_{0,0}\left(w_{1,0}-w_{0,1}\right)=0,
$$

while the other equation becomes

$$
b_{0}+\frac{w_{1,1}}{w_{1,0}-w_{0,1}}\left(\frac{P w_{1,0} z_{0,1}}{w_{1,1} z_{0,1}-1}-\frac{Q w_{0,1} z_{1,0}}{w_{1,1} z_{1,0}-1}\right)-w_{0,0} z_{1,1}=0 .
$$

Note that in both cases the equation pair is still quadrilateral.

In order to derive one-component forms one can use the method of Figure 5, after some modifications in the equations.

C3 in terms of $z$ or $y$ from (3.14): Eliminating variable $y$ leads to (3.5) but in terms of $z$ and $n, m$ reversed.

In order to eliminate $z$ one should first eliminate $z_{0,0}$ from (3.14b) using (3.14a), after which the method of Figure 5 works and yields (3.5) in terms of $y$. 
C3 in terms of $w$ or $z$ from (3.18): After eliminating $z$ one gets (3.5), but now in terms of $w$ and with $(p, q) \mapsto\left(p-b_{0}, p-b_{0}\right)$.

In order to eliminate $w$ one should first eliminate $w_{1,1}$ from (3.14b) using (3.14a), and then one gets (3.5) in terms of $z$ but $n, m$ reversed.

\section{Summary of one-component forms:}

- A2 has two one-component forms: (3.6) in $w=y / x$ and in $z$, and (3.5) in $x$. They correspond to regular and modified BSQ, respectively.

- C3 has two one-component forms: (3.13) in $x$ and (3.5) in $y$ or $w=y / x$ or $z$, corresponding to Schwarzian and modified BSQ, respectively.

Thus we can say that the three-component form of A2 contains both regular and modified BSQ and the three-component form of C3 contains both Schwarzian and modified BSQ. $\mathrm{B} 2$ on the other hand contains only regular BSQ, but in a generalized form in comparison to A2. It is an interesting open question whether there is a three-component version which contains all three different BSQ equations in full generality.

\section{Continuum limits}

We will now consider (semi-)continuous limits of the derived one-component equations: (3.5) for $\mathrm{A} 2$, (3.10) for $\mathrm{B} 2$, and (3.13) for $\mathrm{C}_{b_{0}}$. These are defined on a $3 \times 3$ stencil, see Figure 7.

The technical aspects of taking semi-continuous and fully continuous limits involve several choices, including the gauge (or background solution) and the way the lattice parameters behave under these limits. For example, we know that $p, q$ are related to lattice spacing, but should we let, for example, $p \rightarrow 0$ or $p \rightarrow \infty$ ? Before going into specific equations we are going to discuss some aspects that apply to all cases.

\subsection{Common features}

\subsubsection{Approaching continuum}

The semi-continuous limit means that in some direction the lattice points approach each other to form a continuum. There are two simple ways to squeeze the $3 \times 3$ stencil onto a line, the straight and the skewed way, see Figure 7,1 and 7,2, respectively. (Squeezing in other directions are also possible but the result would depend on still more points and probably would not be useful for applications.)

Before taking the limit we must anchor the discrete and continuous variables. First of all we shift the equations so that the center of the $3 \times 3$ stencil is at $(0,0)$. For the straight limit in the $m$ direction, we can take $m=0$ to correspond to $\xi=0$ and then generically the discrete variable $m$ corresponds to continuous variable $\xi$ and we can write

$$
u_{n, \mu}=u_{n}(\xi+\epsilon \mu)
$$

where $\epsilon$ measures the distance between two lattice points in the $m$ direction. In practice $\mu \in\{-1,0,1\}$. For the skew limit we must take instead

$$
x_{\nu, \mu}=u_{\nu+\mu}\left(\tau+\frac{1}{2}(\nu-\mu) \epsilon\right),
$$




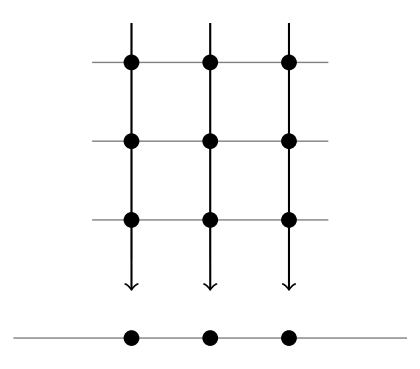

Figure 7.1

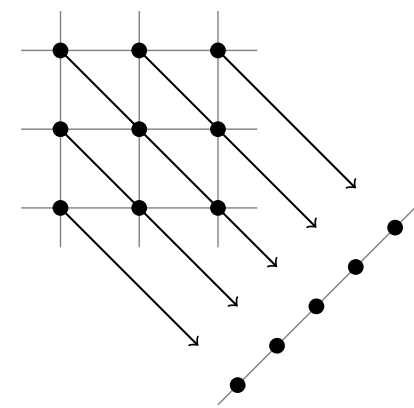

Figure 7 ,2

Figure 7: Two ways to project the $3 \times 3$ stencil to a line: Figure 7,1 gives the straight limit, Figure 7,2 the skew limit. A $90^{\circ}$ degree rotation is also possible.

where $\nu, \mu \in\{-1,0,1\}$.

Now that the dependence on $\epsilon$ is given we can expand in $\epsilon$,

$$
u_{n}(\xi+\epsilon s)=u_{n}(\xi)+\epsilon s u_{n}^{\prime}(\xi)+\frac{1}{2} \epsilon^{2} s^{2} u_{n}^{\prime \prime}(\xi)+\cdots
$$

\subsubsection{Parameter relations}

The above discussion was about limits in general, but in concrete cases we must first determine the connections between the various parameters in the equation and the lattice spacing. One method to get information about this is to linearize the discrete equation and study its discrete plane wave solutions or plane wave factors (PWF) (cf. [26, 33]) Once the PWF is known one can figure out what should be done in order to get, as a limit, the continuous PWF. This is described in Chapter 5 of [16]. In general terms the discrete-continuous relation is an application of

$$
\lim _{n \rightarrow \infty}\left(1+\frac{\alpha}{n}\right)^{n}=e^{\alpha} .
$$

Before one can linearize a nonlinear equation it is necessary to choose a background solution (or gauge) around which to expand. For equation (3.5) we must choose a multiplicative gauge

$$
x_{n, m}=(p-a)^{-n+n_{0}}(q-b)^{-m+m_{0}} c_{1}\left(1+\epsilon v_{n, m}+\cdots\right),
$$

while for (3.10) we must choose additive gauge, such as

$$
x_{n, m}=\sigma\left(n-n_{0}\right)(p-a)+\sigma\left(m-m_{0}\right)(q-b)+\epsilon u_{n, m}+\cdots,
$$

but for (3.13) no gauge is necessary.

We have used here $\left(n_{0}, m_{0}\right)$ as origin but in the expansion these should drop out.

For each of the considered DBSQ equations one finds for $u_{n, m}$ or $v_{n, m}$ the PWF solution:

$$
\text { PWF: } \quad\left(\frac{p-\omega(k)}{p-k}\right)^{n}\left(\frac{q-\omega(k)}{q-k}\right)^{m} \text {, }
$$

where $\omega(k) \neq k$ is one of the roots of $g_{3}(\omega)-g_{3}(k)=0$, where $g_{3}$ is a cubic polynomial, such as (2.29b). 
Straight limit: In order to use (4.4) for a straight limit in the $m$-direction we write

$$
\left(\frac{q-\omega(k)}{q-k}\right)^{m}=\left(1+\frac{k-\omega(k)}{q-k}\right)^{m}
$$

and therefore $q$ must approach infinity as $m$, i.e., $m / q=\xi$. Then

$$
\left(\frac{q-\omega(k)}{q-k}\right)^{m}=\left(1+\frac{\xi(k-\omega(k))}{m-\xi k}\right)^{m} \longrightarrow \exp [\xi(k-\omega(k))], \quad \text { as } m \rightarrow \infty .
$$

Sometimes we may need higher order corrections to the above as follows:

$$
\left(\frac{q-\omega(k)}{q-k}\right)^{m}=\exp \left[\xi(k-\omega(k))+\frac{1}{2}\left(k^{2}-\omega(k)^{2}\right) \xi / q+O\left(1 / q^{2}\right)\right] .
$$

These results suggest that in a straight limit in $m$ direction we should take

$$
m=\xi q, \quad q \rightarrow \infty
$$

Skew limit: In the skew limit, as in Figure 7,2, we first need to make a $45^{\circ}$ degree rotation, and after defining $N=n+m, m^{\prime}=\frac{1}{2}(n-m)$, i.e., $n=\frac{1}{2} N+m^{\prime}, m=\frac{1}{2} N-m^{\prime}$, we get?

$$
\begin{aligned}
& \left(\frac{p-\omega(k)}{p-k}\right)^{n}\left(\frac{q-\omega(k)}{q-k}\right)^{m} \\
= & \left(\frac{p-\omega(k)}{p-k} \cdot \frac{q-\omega(k)}{q-k}\right)^{N / 2}\left(\frac{p-k}{p-\omega(k)} \cdot \frac{q-\omega(k)}{q-k}\right)^{m^{\prime}} \\
= & \left(\frac{p-\omega(k)}{p-k} \cdot \frac{q-\omega(k)}{q-k}\right)^{N / 2}\left(1+\frac{(k-\omega(k))(p-q)}{(p-\omega(k))(q-k)}\right)^{m^{\prime}} .
\end{aligned}
$$

This suggests that $p-q$ should approach 0 , thus we take

$$
q=p-\delta, \quad \tau=\delta m^{\prime}, \quad \delta \rightarrow 0
$$

and then the expansion yields

$$
\left(1+\frac{(k-\omega(k)) \delta}{(p-\omega(k))(p-k-\delta)}\right)^{\tau / \delta}=\exp \left[\frac{(k-\omega(k)) \tau}{(p-\omega(k))(p-k)}+O\left(\delta^{2}\right)\right] .
$$

Thus the main results we have so far obtained from PWF analysis are the limit behaviors (4.8) and (4.9) for straight and skew limits, respectively. Expanding in higher orders will be useful in deriving double limits.

Finally we must relate the lattice parameters $p, q$ used in the limits to the parameters $P, Q$ appearing in the equation, we use

$$
P=g_{3}(p) \text { or } P=g_{3}(p)-g_{3}(a), \text { where } g_{3}(x):=x^{3}+\alpha_{2} x^{2}+\alpha_{1} x+\alpha_{0},
$$

and similarly for $Q$. (This $g_{3}$ is a generalized version of (2.29b) with additional $\alpha_{j}$.) The cubic term is a signature of Boussinesq equations. It should also be noted that as far as the CAC test is concerned, the parameters $P, Q$ can be arbitrary. However, if we want the discrete equation to have a continuum limit to a (semi-)continuous Boussinesq equation then the cubic form is necessary, furthermore $P, Q$ may depend on some other parameters of the equation.

\footnotetext{
${ }^{3}$ Note that one can also take $N=n+m, m^{\prime}=m$, cf. [16].
} 


\section{$4.2 \quad$ B2}

As the first case we take equation (3.10), but centered at the origin

$$
\begin{aligned}
(P-Q)( & \left.\frac{1}{x_{1,-1}-x_{0,0}}-\frac{1}{x_{0,0}-x_{-1,1}}\right)+b_{0}\left(x_{-1,0}-x_{0,-1}+x_{1,0}-x_{0,1}\right) \\
& \quad-\left(x_{1,1}-x_{-1,0}\right)\left(x_{1,0}-x_{0,1}\right)-\left(x_{-1,-1}-x_{1,0}\right)\left(x_{0,-1}-x_{-1,0}\right)=0 .
\end{aligned}
$$

\subsubsection{Straight limit}

We use the linear background $x=p n+q m+\epsilon u$ and take $\epsilon=1 / q \rightarrow 0$ with initially arbitrary $P, Q$ as in (4.10). Then using (4.1) and expanding we find from order 0 that $\alpha_{3}=1$, and at order 1 that $\alpha_{2}=-b_{0} 4$ Finally (if $\alpha_{1}=0$, in agreement with (2.29b)) at order 2 we get the semi-discrete three-point equation

$$
\begin{aligned}
& u_{1}^{\prime \prime}+u_{0}^{\prime \prime}+u_{-1}^{\prime \prime}-3 u_{1}^{\prime}\left(p-u_{0}+u_{1}\right)+3 u_{-1}^{\prime}\left(p-u_{-1}+u_{0}\right) \\
& +\left(p-u_{0}+u_{1}\right)^{3}-\left(p-u_{-1}+u_{0}\right)^{3} \\
& -b_{0}\left[u_{-1}^{\prime}-u_{1}^{\prime}+\left(2 p-u_{-1}+u_{1}\right)\left(u_{-1}-2 u_{0}+u_{1}\right)\right]=0 .
\end{aligned}
$$

where the primes refer to derivatives with respect to $\xi$, cf. (4.1). Note that when $b_{0}=0$, the above equation is Eq.(5.9.13) in [37] (with $u \rightarrow-u$ ). The $b_{0}$ term is actually a discrete derivative of the semi-discrete $\mathrm{lpKdV}$ equation

$$
u_{0}^{\prime}+u_{1}^{\prime}+\left(2 p-u_{0}+u_{1}\right)\left(u_{0}-u_{1}\right)=0 .
$$

\subsubsection{Skew limit}

As discussed before, we should make a $45^{\circ}$ degree rotation and then take the continuous limit in the $m^{\prime}$ direction. We use the same linear background as before, and the form of $P, Q$ found for the straight limit, but instead of (4.8) and (4.1) we use (4.9) and (4.2).

Then at order $\epsilon^{3}$ we get the following five-point equation

$$
\begin{aligned}
\left(3 p^{2}-2 p b_{0}\right) \ddot{u}_{0}+\left(\dot{u}_{0}+1\right)^{2}[ & \left(u_{2}-u_{-1}-b_{0}+3 p\right)\left(\dot{u}_{1}+1\right) \\
& \left.+\left(u_{-2}-u_{1}+b_{0}-3 p\right)\left(\dot{u}_{-1}+1\right)\right]=0,
\end{aligned}
$$

where the dot refers to derivatives with respect to $\tau$ in (4.2) . For $b_{0}=0$ this agrees with (5.9.4) in [37], up to $p \rightarrow-p$.

\subsubsection{Double limit}

Now we take equation (4.11) and do the straight continuum limit on the remaining discrete variable using $p=1 / \delta$. We set $u_{\nu}(\xi)=v(\tau+\nu \delta, \xi)$ and apply Taylor expansion as in (4.3) and the leading term in the expansion would then be $\left(\partial_{\xi}-\partial_{\tau}\right) v(\tau, \xi)=0$, which means that the naive limit does not work. In order to get a better understanding of the situation we return to the PWF (4.7). With $q \rightarrow \infty$ limit already taken we expand in $1 / p$ :

$$
\left(\frac{p-\omega(k)}{p-k}\right)^{n} e^{(k-\omega(k)) \xi}=\exp \left[(k-\omega(k))(\xi+\tau)+\frac{1}{2}\left(k^{2}-\omega^{2}(k)\right) \frac{\tau}{p}+O\left(\frac{1}{p^{2}}\right)\right], \tau=\frac{n}{p} .
$$

\footnotetext{
${ }^{4}$ This means in particular that although $P, Q$ and $b_{0}$ were independent in the CAC analysis, they must be related in the indicated way before we have a reasonable continuum limit.
} 
This suggests that we should introduce new variables

$$
x=\xi+\tau, \quad t=\tau / p
$$

which means

$$
\partial_{\xi}=\partial_{x}, \quad \partial_{\tau}=\partial_{x}+\frac{1}{p} \partial_{t},
$$

and apply these in the expansion, before actually taking the limit $p \rightarrow \infty$. In other words, the variables $\xi$ and $\tau$ are infinitesimally close and therefore the leading term combines with some higher order terms. With this modification the leading term becomes

$$
v_{x x x x}-12 v_{x x} v_{x}-8 b_{0} v_{x t}+12 v_{t t}=0 .
$$

The $v_{x t}$ term can be converted into $v_{x x}$ by the translation $\partial_{t} \mapsto \partial_{t}+\frac{1}{3} b_{0} \partial_{x}$ (which could have been be added into (4.15) ) which yields

$$
v_{x x x x}-12 v_{x x} v_{x}-\frac{4}{3} b_{0}^{2} v_{x x}+12 v_{t t}=0 .
$$

This is the standard Boussinesq equation with a mass term.

We can also take the double continuum limit by starting from (4.12), where the continuous variable is $\tau$ and $p=1 / \delta$. Thus we expand $u_{\nu}(\tau)=v(\xi+\nu \delta, \tau)$, but a change of the continuous variables is also necessary. If we define $x, t$ by

$$
\partial_{\tau}=\left[1+\delta \frac{1}{3} b_{0}\right] \delta^{2} \partial_{x}+\delta^{3} \partial_{t}, \quad \partial_{\xi}=\partial_{x},
$$

we get (4.16).

Finally, we can take a double limit directly from the fully discrete form (3.10). For that purpose we take the linear gauge as before, and the limit by $p=p_{0} / \epsilon, q=q_{0} / \epsilon, \epsilon \rightarrow 0$ with

$$
u_{n, m}=-v\left(x+n A+m B, y+(n+m) \epsilon^{2} /\left(p_{0} q_{0}\right)\right)
$$

where

$$
A:=-\epsilon / p_{0}-\left(\epsilon^{2} b_{0}\right) /\left(3 p_{0}^{2}\right), \quad B:=-\epsilon / q_{0}-\left(\epsilon^{2} b_{0}\right) /\left(3 q_{0}^{2}\right) .
$$

After expanding in $\epsilon$ the leading term is again (4.16).

\section{$4.3 \quad \mathrm{~A} 2$}

Now we consider equation (3.5), centered at the origin:

$$
\left(\frac{P x_{0,0}-Q x_{-1,1}}{x_{-1,1}-x_{0,0}}\right) \frac{x_{0,1}}{x_{-1,0}}-\left(\frac{P x_{1,-1}-Q x_{0,0}}{x_{0,0}-x_{1-1}}\right) \frac{x_{1,0}}{x_{0,-1}}=\frac{x_{-1,-1}}{x_{0,-1}}-\frac{x_{-1,-1}}{x_{-1,0}}-\frac{x_{0,1}}{x_{1,1}}+\frac{x_{1,0}}{x_{1,1}} .
$$

In this case we use the gauge transformation (4.5) and the cubic parameterization (4.10).

\subsubsection{Straight limit}

As in Section 4.2.1 we use (4.8), (4.10) and (4.1) but now with a multiplicative gauge (4.5) and take $\alpha_{3}=\sigma=1, b=a$ after which the leading term in the expansion will yield 
a second order three-point equation:

$$
\begin{aligned}
& \frac{v_{1}^{\prime \prime}}{v_{1}}+\frac{v_{0}^{\prime \prime}}{v_{0}}+\frac{v_{-1}^{\prime \prime}}{v_{-1}}-2\left(\frac{v_{1}^{\prime}}{v_{1}}\right)^{2}-\left(\frac{v_{0}^{\prime}}{v_{0}}\right)^{2}-\frac{v_{1}^{\prime} v_{0}^{\prime}}{v_{1} v_{0}}+\frac{v_{-1}^{\prime} v_{0}^{\prime}}{v_{-1} v_{0}} \\
& \quad+3(p-a)\left(\frac{v_{-1}^{\prime}}{v_{0}}-\frac{v_{1}^{\prime} v_{0}}{v_{1}^{2}}\right)+\left(\alpha_{2}+3 a\right)\left(\frac{v_{-1}^{\prime}}{v_{-1}}-\frac{v_{1}^{\prime}}{v_{1}}\right)-\left(\frac{v_{1}}{v_{0}}-\frac{v_{0}}{v_{-1}}\right) \frac{P(p)}{a-p} \\
& \quad-\left(\frac{v_{-1}}{v_{0}}-\frac{v_{0}}{v_{1}}\right)\left(\alpha_{2}+3 a\right)(a-p)+\left(\frac{v_{-1}^{2}}{v_{0}^{2}}-\frac{v_{0}^{2}}{v_{1}^{2}}\right)(a-p)^{2}=0 .
\end{aligned}
$$

This can also be written as

$$
\begin{aligned}
{\left[\ln \left(v_{-1} v_{0} v_{1}\right)\right]^{\prime \prime} } & +\left(\ln v_{-1}-\ln v_{1}\right)^{\prime}\left[\alpha_{2}+3 a+\left(\ln \left(v_{-1} v_{0} v_{1}\right)\right)^{\prime}\right] \\
& +(p-a)\left[\frac{v_{-1}}{v_{0}}\left(\alpha_{2}+3 a+3\left(\ln v_{-1}\right)^{\prime}\right)-\frac{v_{0}}{v_{1}}\left(\alpha_{2}+3 a+3\left(\ln v_{1}\right)^{\prime}\right)\right] \\
& +\frac{P(p)}{p-a}\left(\frac{v_{1}}{v_{0}}-\frac{v_{0}}{v_{-1}}\right)+(p-a)^{2}\left(\frac{v_{-1}^{2}}{v_{0}^{2}}-\frac{v_{0}^{2}}{v_{1}^{2}}\right)=0 .
\end{aligned}
$$

If $\alpha_{2}=a=0$ this is the same as (5.9.14) in [37.

\subsubsection{Skew limit}

For the skew limit we also use multiplicative gauge (4.5) and expansion (4.2) with (4.9) and cubic parameterization. The semi-continuous limit is then

$$
\partial_{\tau}\left(\frac{v_{1}}{v_{-1}} \frac{\Pi_{1} \dot{v}_{0}+\Pi_{2} v_{0}}{(p-a) \dot{v}_{0}-v_{0}}\right)-\frac{v_{-2}}{v_{-1}}\left((p-a) \frac{\dot{v}_{-1}}{v_{-1}}-1\right)+\frac{v_{1}}{v_{2}}\left((p-a) \frac{\dot{v}_{1}}{v_{1}}-1\right)=0
$$

where

$$
\begin{aligned}
& \Pi_{1}=\left(g_{3}(p)-g_{3}(a)\right) /(a-p), \\
& \Pi_{2}=-\left(a+2 p+\alpha_{2}\right) .
\end{aligned}
$$

\subsubsection{Double limit}

Again we can derive the fully continuous limit from either the straight semi-continuous equation (4.19) or from the skew semi-continuous limit (4.21) or taking a double limit directly from (3.5). For A2 there is the special feature that in order to reach the modified BSQ equation we must also change the dependent variables by $v=e^{V}$.

Starting with the straight semi-continuous limit (4.19) we use again $p=1 / \delta$ and $u_{\nu}=v(y+\nu \delta, x), v=e^{V}$ and furthermore we use the change of variables 5

$$
\partial_{y}=\left(1-\frac{1}{3} \alpha_{2} \delta\right) \partial_{x}+\frac{1}{2} \delta \partial_{t}
$$

and then we obtain

$$
V_{x x x x}-6 V_{x x} V_{x}^{2}+6 V_{x x} V_{t}-4\left(\alpha_{2}+3 a\right) V_{x x} V_{x}+4\left(\alpha_{1}-\frac{1}{3} \alpha_{2}^{2}\right) V_{x x}+3 V_{t t}=0 .
$$

This is the modified Boussinesq equation (cf. [21], Equation (3.5)).

\footnotetext{
${ }^{5}$ This transformation is needed in order to eliminate the cross term $V_{x t}$ in favor of the mass term $V_{x x}$.
} 
From the skew limit (4.21) we use $p=1 / \delta, \alpha_{3}=1$ and the variable change

$$
\partial_{y}=\delta^{2}\left(-1+\frac{1}{3} \alpha_{2} \delta\right) \partial_{x}-\delta^{3} \partial_{t},
$$

and get the same result (4.22).

Finally the double limit directly from (3.5) is taken with (4.17) except now

$$
A:=\epsilon / p_{0}-\left(\epsilon / p_{0}\right)^{2} \frac{1}{3} \alpha_{2}, \quad B:=\epsilon / q_{0}-\left(\epsilon / q_{0}\right)^{2} \frac{1}{3} \alpha_{2} .
$$

The result is again the same as in (4.22).

\section{$4.4 \quad \mathrm{C} 3$}

We consider next the continuum limits of (3.13), centered at $(0,0)$ :

$$
\begin{aligned}
& \frac{\left(x_{1,1}-x_{0,1}\right)\left(x_{-1,1}-x_{0,0}\right)\left(x_{-1,0}-x_{-1,-1}\right)}{\left(x_{1,1}-x_{1,0}\right)\left(x_{0,0}-x_{1,-1}\right)\left(x_{0,-1}-x_{-1,-1}\right)}= \\
& \frac{\left(x_{0,0}-x_{-1,1}\right)\left(b_{0} x_{-1,0}+b_{1}\right)+\left(x_{0,1}-x_{-1,1}\right)\left(x_{-1,0}-x_{0,0}\right) P-\left(x_{0,1}-x_{0,0}\right)\left(x_{-1,0}-x_{-1,1}\right) Q}{\left(x_{1,-1}-x_{0,0}\right)\left(b_{0} x_{0,-1}+b_{1}\right)+\left(x_{1,0}-x_{0,0}\right)\left(x_{0,-1}-x_{1,-1}\right) P-\left(x_{1,0}-x_{1,-1}\right)\left(x_{0,-1}-x_{0,0}\right) Q} .
\end{aligned}
$$

\subsubsection{Straight limit}

We use the usual straight limit approach (4.1), (4.8), and (4.10) but without any gauge transformation. The leading term in the expansion is then

$$
\begin{gathered}
\partial_{x} \log \left[P(p)\left(u_{1}-u_{-1}\right)-b_{1}-b_{0} u_{-1}+\frac{u_{1}^{\prime} u_{0}^{\prime} u_{-1}^{\prime}}{\left(u_{1}-u_{0}\right)\left(u_{0}-u_{-1}\right)}\right] \\
=\frac{u_{1}^{\prime}}{u_{1}-u_{0}}-\frac{u_{-1}^{\prime}}{u_{0}-u_{-1}} .
\end{gathered}
$$

A special case of this appears in [37] (5.9.15).

\subsubsection{Skew limit}

For the skew limit we use (4.2), (4.9) and (4.10), without gauge transform, and the leading term in the expansion yields

$$
\begin{gathered}
\partial_{\tau} \log \left[P(p)\left(u_{1}-u_{-1}\right)-b_{1}-b_{0} u_{-1}+\frac{\left(3 p^{2}+2 \alpha_{2} p+\alpha_{1}\right)\left(u_{1}-u_{0}\right)\left(u_{0}-u_{-1}\right)}{\dot{u}_{0}}\right] \\
=\frac{\dot{u}_{-1}}{u_{-1}-u_{-2}}-\frac{\dot{u}_{1}}{u_{2}-u_{1}} .
\end{gathered}
$$

This has some similarity to the straight limit. When $P(p)=p^{3}, b_{1}=b_{0}=0$, one will get (5.9.9) in [37] (up to signs). 


\subsubsection{Double limit}

Starting from (4.24) and using $p=1 / \delta$ and $u_{\nu}=v(y+\nu \delta, x)$ with the additional variable change defined by

$$
\partial_{y}=\left(1-\frac{1}{3} \alpha_{2} \delta\right) \partial_{x}+\frac{1}{2} \delta \partial_{t}
$$

we obtain

$$
3 \partial_{t}\left(\frac{v_{t}}{v_{x}}\right)+\partial_{x}\left(\frac{v_{x x x}}{v_{x}}+\frac{3}{2} \frac{v_{t}^{2}-v_{x x}^{2}}{v_{x}^{2}}-4 \frac{b_{1}+b_{0} v}{v_{x}}\right)=0 .
$$

The double limit obtained by starting from the skew semi-continuous limit (4.25), together with variable change

$$
\partial_{y}=\left(-1+\frac{1}{3} \alpha_{2} \delta\right) \delta^{2} \partial_{x}+\frac{1}{2} \delta^{3} \partial_{t}
$$

again yields (4.26).

The direct double limit from (3.13) using (4.17) with (4.23), but with the second variable in (4.17) given by $y-(n+m) \epsilon^{2} /\left(2 p_{0} q_{0}\right)$, leads again to (4.26), except for $b_{1} \mapsto-b_{1}$.

If $b_{0}=b_{1}=0$ in (4.26) it is the Schwarzian BSQ (see, e.g., [38] Eq. (4.9)), however the additional $b_{0}, b_{1}$ terms break the Möbius invariance.

\section{$5 \quad$ Lax pairs}

In general Lax pairs can be generated from CAC: One takes the side equations and interprets the bar-shifted variables as linear Lax variables. However, the equations defined on an edge only are not convenient, thus one usually takes some linear combinations of them. The construction of Lax matrices using CAC can be automatized to some extent, see [3] 5 ].

One important requirement for the Lax matrices is that they should contain a spectral parameter, in the following it will be $R$.

\section{$5.1 \quad$ B2}

The B2 equations were given (2.3). On the left side of the cube we then have

$$
\begin{aligned}
& \widetilde{y}=x \widetilde{x}-z, \quad \bar{y}=x \bar{x}-z, \\
& \overline{\widetilde{y}}=\overline{\bar{x}} \overline{\bar{x}}-\bar{z}, \quad \overline{\widetilde{y}}=\widetilde{x} \overline{\widetilde{x}}-\widetilde{z},
\end{aligned}
$$

and if from the second set we eliminate $\overline{\widetilde{y}}$ or $\overline{\widetilde{x}}$ we get

$$
\overline{\widetilde{x}}=\frac{\widetilde{z}-\bar{z}}{\widetilde{x}-\bar{x}}, \quad \overline{\widetilde{y}}=\frac{\widetilde{z} \bar{x}-\bar{z} \widetilde{x}}{\widetilde{x}-\bar{x}} .
$$

Now we introduce [4]

$$
\bar{x}=\frac{\phi_{1}}{\phi_{0}}, \quad \bar{z}=\frac{\phi_{2}}{\phi_{0}}, \quad \bar{y}=\frac{\phi_{3}}{\phi_{0}},
$$

and then the equations in (5.3) can be written as

$$
\begin{aligned}
\widetilde{\phi}_{0} & =\gamma\left(\widetilde{x} \phi_{0}-\phi_{1}\right), \\
\widetilde{\phi}_{1} & =\gamma\left(\widetilde{z} \phi_{0}-\phi_{2}\right), \\
\widetilde{\phi}_{3} & =\gamma\left(\widetilde{z} \phi_{1}-\widetilde{x} \phi_{2}\right),
\end{aligned}
$$


where $\gamma$ is the separation factor. For the $\overline{\widetilde{z}}$ equation we take tilde-bar version of (2.3c) (with also $Q \rightarrow R$ ) and eliminate from it $\overline{\widetilde{x}}$ using (5.3), this results with

$$
\begin{aligned}
\widetilde{\phi}_{2} & =\gamma\left(A_{p} \phi_{0}+\left(b_{0} x-b_{1}+y\right) \phi_{1}-\left(b_{0}+x\right) \phi_{2}\right), \\
\text { where } \quad & A_{p}:=\left(b_{1}-b_{0} x-y\right) \widetilde{x}+\left(b_{0}+x\right) \widetilde{z}+P-R .
\end{aligned}
$$

The above can also be done for the hat-bar version. We then get the matrix equations

$$
\widetilde{\phi}=\boldsymbol{L} \phi, \quad \widehat{\phi}=\boldsymbol{M} \phi,
$$

in which [4]

$$
\phi=\left(\begin{array}{c}
\phi_{0} \\
\phi_{1} \\
\phi_{2} \\
\phi_{3}
\end{array}\right), \quad \boldsymbol{L}_{4 \times 4}^{B 2}=\gamma\left(\begin{array}{cccc}
\widetilde{x} & -1 & 0 & 0 \\
\widetilde{z} & 0 & -1 & 0 \\
A_{p} & y+b_{0} x-b_{1} & -b_{0}-x & 0 \\
0 & \widetilde{z} & -\widetilde{x} & 0
\end{array}\right) .
$$

The matrix $\boldsymbol{M}$ is the hat- $q$ version of $\boldsymbol{L}$. Now the last column of $\boldsymbol{L}, \boldsymbol{M}$ is a null column and therefore we can reduce the system to a $3 \times 3$ matrix problem with

$$
\phi=\left(\begin{array}{c}
\phi_{0} \\
\phi_{1} \\
\phi_{2}
\end{array}\right), \quad \boldsymbol{L}_{3 \times 3}^{B 2}=\gamma\left(\begin{array}{ccc}
\widetilde{x} & -1 & 0 \\
\widetilde{z} & 0 & -1 \\
A_{p} & y+b_{0} x-b_{1} & -b_{0}-x
\end{array}\right) .
$$

The compatibility condition following from (5.6) is

$$
\widehat{L} \boldsymbol{M}=\widetilde{M} L .
$$

For this equation it would be best to choose the separation factor $\gamma$ so that $\operatorname{det} \boldsymbol{L}=$ const., because then part of the matrix equation is immediately satisfied by taking determinants of both sides. In this case we can take $\gamma=1$ because $\operatorname{det} \boldsymbol{L}=P-R$. With this choice (5.9) yields three equations:

$$
\begin{aligned}
& \widehat{\widetilde{x}}=\frac{\widetilde{z}-\widehat{z}}{\widetilde{x}-\widehat{x}}, \quad x=\frac{\widetilde{y}-\widehat{y}}{\widetilde{x}-\widehat{x}}, \\
& \widehat{\widetilde{z}}=b_{0}(\widehat{\widetilde{x}}-x)+x \widehat{\widetilde{x}}+b_{1}-y+\frac{P-Q}{\widetilde{x}-\widehat{x}}
\end{aligned}
$$

This is not completely equivalent to (2.3) because we do not have un-shifted $z$, nor $\widetilde{z}$ and $\widehat{z}$ separately.

In the language of DLA version of B2 (2.28) (with $b_{1}=0$ ), the $3 \times 3$ Lax matrices are as follows,

$$
\boldsymbol{L}^{B 2 D}=\left(\begin{array}{ccc}
p-\widetilde{u}^{(0,0)} & 1 & 0 \\
-\widetilde{u}^{(1,0)} & p-b_{0} & 1 \\
* & -u^{(0,1)}-2 b_{0} u^{(0,0)}-2 b_{0}^{2} & p+2 b_{0}+u^{(0,0)}
\end{array}\right)
$$

in which $*=R-P-\left(p-\widetilde{u}^{(0,0)}\right)\left[\left(p+b_{0}\right)\left(p+u^{(0,0)}\right)+u^{(0,1)}\right]-\left(p+u^{(0,0)}+2 b_{0}\right) \widetilde{u}^{(1,0)}$,

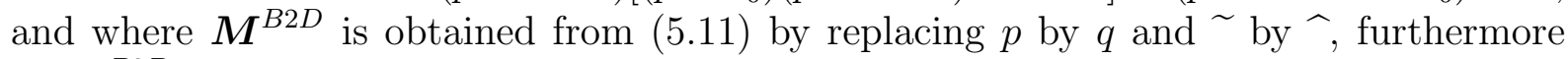
$\operatorname{det}\left[\boldsymbol{L}^{B 2 D}\right]=R-P$. The compatibility leads to the equations

$$
\widehat{u}^{(1,0)}-\widetilde{u}^{(1,0)}=\left(p-q+\widehat{u}^{(0,0)}-\widetilde{u}^{(0,0)}\right) \widehat{\widetilde{u}}^{(0,0)}-p \widehat{u}^{(0,0)}+q \widetilde{u}^{(0,0)}
$$


and

$$
\widehat{u}^{(0,1)}-\widetilde{u}^{(0,1)}=\left(p-q+\widehat{u}^{(0,0)}-\widetilde{u}^{(0,0)}\right) u^{(0,0)}-p \widetilde{u}^{(0,0)}+q \widehat{u}^{(0,0)},
$$

together with an equation that is equivalent to equation (2.28c), if we use $\widehat{\widetilde{u}}^{(0,0)}$ as solved from the first equation and $u^{(0,0)}$ solved from the second.

The B2 Lax pair was first given in [27] with $\alpha_{1}=\alpha_{2}=b_{0}=b_{1}=0$ and in [41] with full parameterization.

\section{$5.2 \quad \mathrm{~A} 2$}

From CAC: The A2 equation was given in (2.4), where we can take $b_{0}=0$. Using (2.4a) and the bar-version of (2.4b) and their shifts, we can easily derive

$$
\overline{\widetilde{x}}=\frac{\widetilde{x}-\bar{x}}{\widetilde{z}-\bar{z}}, \quad \overline{\widetilde{y}}=\frac{\widetilde{x} \bar{z}-\bar{x} \widetilde{z}}{\widetilde{z}-\bar{z}}
$$

To these and to the tilde-bar version of (2.4c) we use (15.4) and obtain the Lax matrix [41]

$$
\boldsymbol{L}=\gamma(x, \widetilde{x})\left(\begin{array}{cccc}
\widetilde{z} & 0 & -1 & 0 \\
\widetilde{x} & -1 & 0 & 0 \\
\frac{y \widetilde{z}}{x}-\frac{P \widetilde{x}}{x} & \frac{R}{x} & -\frac{y}{x} & 0 \\
0 & -\widetilde{z} & \widetilde{x} & 0
\end{array}\right)
$$

Again the last column vanishes and we use instead an invertible $3 \times 3$ matrix [41]

$$
\boldsymbol{L}^{A 2}=\gamma(x, \widetilde{x})\left(\begin{array}{ccc}
\widetilde{z} & 0 & -1 \\
\widetilde{x} & -1 & 0 \\
\frac{y \widetilde{z}}{x}+\frac{P \widetilde{x}}{x} & -\frac{R}{x} & -\frac{y}{x}
\end{array}\right),
$$

and the matrix $\boldsymbol{M}^{A 2}$ is the hat- $q$ version of $\boldsymbol{L}^{A 2}$. If we again normalize the Lax matrix by the condition $\operatorname{det}[\boldsymbol{L}]=$ const, we should take $\gamma(a, b)=(a / b)^{1 / 3}$. Then the compatibility condition (5.9) yields

$$
\widehat{\widetilde{x}}=\frac{\widetilde{x}-\widehat{x}}{\widetilde{z}-\widehat{z}}, \quad x=\frac{\widehat{x} \widetilde{y}-\widetilde{x} \widehat{y}}{\widetilde{x}-\widehat{x}}
$$

together with eq. (2.4c).

From DLA: In the DLA the A2 equation is given in (2.33) and the Lax matrix given there is 41 .

$$
\boldsymbol{L}^{A 2}=\left(\begin{array}{ccc}
p-a & \widetilde{v}_{a} & 0 \\
0 & p-\widetilde{u}^{(0,0)} & 1 \\
\frac{G_{3}(R,-a)}{v_{a}} & * & p-\alpha_{2}+\frac{s_{a}}{v_{a}}
\end{array}\right)
$$

in which $*=\left(p-\widetilde{u}^{(0,0)}\right)\left(p-\alpha_{2}+s_{a} / v_{a}\right)-p_{a} \widetilde{v}_{a} / v_{a}, R$ stands for the spectral parameter, and

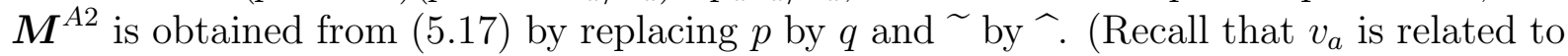
$x, u^{(0,0)}$ to $z$ and $s_{a}$ to $y$.) The Lax compatibility condition leads to the equations (2.33c) and

$$
\begin{aligned}
& p-q+\widehat{u}^{(0,0)}-\widetilde{u}^{(0,0)}=(p-a) \frac{\widehat{v}_{a}}{\widehat{\widehat{v}}_{a}}-(q-a) \frac{\widetilde{v}_{a}}{\widehat{\widehat{v}}_{a}} \\
& p-q+\frac{\widehat{s}_{a}}{\widehat{v}_{a}}-\frac{\widetilde{s}_{a}}{\widetilde{v}_{a}}=(p-a) \frac{v_{a}}{\widetilde{v}_{a}}-(q-a) \frac{v_{a}}{\widehat{v}_{a}} .
\end{aligned}
$$


From the two-component form: The A2 equation has also a two-component form,

$$
\begin{aligned}
& \frac{z-\widetilde{w}}{z-\widehat{w}}=\frac{\widehat{z}-\widehat{\widetilde{w}}}{\widetilde{z}-\widehat{\widetilde{w}}}, \\
& (\widetilde{z}-\widehat{z})(\widehat{\widetilde{z}}-w)=\frac{P}{z-\widetilde{w}}-\frac{Q}{z-\widehat{w}} .
\end{aligned}
$$

This pair is still defined on the elementary square and is 3-dimensionally consistent, but it is not possible to construct a Lax pair using the sides of the consistency cube, because it leads to an expression that is quadratic in the auxiliary field $\phi$. However, a $2 \times 2$ Lax matrix was given in 31] (see eqn. (2.4.5)) (although this does not contain spectral parameter):

$$
\begin{aligned}
\boldsymbol{L}_{2 \times 2}^{A 2} & =\left(\begin{array}{cc}
\widetilde{z} & -1 \\
w \widetilde{z}+\frac{P}{z-\widetilde{w}} & -w
\end{array}\right), \\
\boldsymbol{M}_{2 \times 2}^{A 2} & =\left(\begin{array}{cc}
\widehat{z} & -1 \\
w \widehat{z}+\frac{Q}{z-\widehat{w}} & -w
\end{array}\right),
\end{aligned}
$$

which directly yields (5.19) from the compatibility $\widehat{\boldsymbol{L}} \boldsymbol{M}=\widetilde{\boldsymbol{M}} \boldsymbol{L}$.

Still another Lax matrix generating (5.19) is given in [9] (see page 16)

$$
\boldsymbol{L}^{F X}=\left(\begin{array}{ccc}
\widetilde{z}-w & 0 & -1 \\
-1 & \widetilde{w}-z & 0 \\
\frac{P}{\widetilde{w}-z}-(\widetilde{w}-w)(\widetilde{z}-w) & R & \widetilde{w}-w
\end{array}\right),
$$

with corresponding $\boldsymbol{M}^{F X}$. As mentioned above this Lax pair cannot arise from CAC analysis of the type that worked for the other equations. However, since this Lax matrix resembles some of the other CAC-generated Lax matrices, we could try to reverse engineer and see where it could come from. If we write out the $3 \times 3$ version of $\widetilde{\phi}=\boldsymbol{L}^{F X} \phi$ and then divide the second and third equation by the first we get

$$
\begin{aligned}
& \frac{\widetilde{\phi}_{1}}{\widetilde{\phi}_{0}}=\frac{1+(\widetilde{z}-w) \frac{\phi_{1}}{\phi_{0}}}{w-\widetilde{z}+\frac{\phi_{2}}{\phi_{0}}}, \\
& \frac{\widetilde{\phi}_{2}}{\widetilde{\phi}_{0}}=\frac{R \frac{\phi_{1}}{\phi_{0}}+(\widetilde{w}-w) \frac{\phi_{1}}{\phi_{0}}+\frac{P}{\widetilde{w}-z}-(\widetilde{w}-w)(\widetilde{z}-w)}{w-\widetilde{z}+\frac{\phi_{2}}{\phi_{0}}} .
\end{aligned}
$$

Previously we associated bar-quantities to $\phi$ ratios as in (5.4) but it does not work here. We must instead take

$$
\frac{\phi_{1}}{\phi_{0}}=\frac{1}{\bar{w}-z}, \quad \frac{\phi_{2}}{\phi_{0}}=\bar{z}-w,
$$

and this choice yields (5.19) from (5.22).

\section{$5.3 \quad \mathrm{C} 3$}

The C3 equation (2.5) is

$$
\begin{aligned}
& x-\widetilde{x}=\widetilde{y} z, \quad x-\widehat{x}=\widehat{y} z, \\
& \widehat{\widetilde{z}} y=z \frac{P \widehat{z} \widetilde{y}-Q \widetilde{z} \hat{y}}{\widetilde{z}-\widehat{z}}+b_{1}+b_{0} x .
\end{aligned}
$$


Several other equations can be derived from (5.24a), for example

$$
\widehat{\widetilde{x}}=\frac{\widehat{x} \widetilde{z}-\widetilde{x} \widehat{z}}{\widetilde{z}-\widehat{z}}, \quad x=\frac{\widehat{x} \widetilde{y}-\widetilde{x} \widehat{y}}{\widetilde{y}-\widehat{y}}, \quad z=-\frac{\widetilde{x}-\widehat{x}}{\widetilde{y}-\widehat{y}}, \quad \widehat{\widetilde{y}}=-z \frac{\widetilde{y}-\widehat{y}}{\widetilde{z}-\widehat{z}} .
$$

If we consider the tilde-bar versions of these and take equation for $\overline{\widetilde{x}}$ from (5.25) and equation (5.24b) in which $\widetilde{y}$ and $\bar{y}$ have been eliminated using (5.24a), then we can use the $\mathrm{CAC}$ method and construct the $3 \times 3$ Lax matrix

$$
\boldsymbol{L}_{3 \times 3}^{C 3}=\frac{1}{z}\left(\begin{array}{ccc}
\widetilde{z} & 0 & -1 \\
0 & \widetilde{z} & -\widetilde{x} \\
\frac{\tilde{z}}{y}\left(b_{1}+\left(b_{0}-R\right) x\right) & \frac{\widetilde{z}}{y} R & \frac{1}{y}\left(P(\widetilde{x}-x)-b_{1}-b_{0} x\right)
\end{array}\right) .
$$

The compatibility conditions arising from this Lax matrix and its hat-Q companion yield the equations for $\widehat{\widetilde{x}}$ and $x$ in (5.25). However, for $\widehat{\widetilde{z}}$ it produces an equation that agrees with (5.24b) only after we also use the equation for $z$ in (5.25), which does not follow from Lax compatibility.

There is also the $4 \times 4$ Lax matrix that can be obtained from CAC by adding the $\widehat{\widetilde{y}}$ equation from (15.25):

$$
\boldsymbol{L}_{4 \times 4}^{C 3}=\frac{1}{z}\left(\begin{array}{cccc}
\widetilde{z} & 0 & 0 & -1 \\
0 & \widetilde{z} & 0 & -\widetilde{x} \\
-z \widetilde{y} & 0 & z & 0 \\
\frac{\widetilde{z}}{y}\left(b_{1}+b_{0} x\right) & 0 & -\frac{z \widetilde{z}}{y} R & \frac{1}{y}\left(P z \widetilde{y}-b_{1}-b_{0} x\right)
\end{array}\right),
$$

and the matrix $\boldsymbol{M}_{4 \times 4}^{C 3}$ is the hat- $Q$ version. In this case the compatibility conditions yield equation (5.24b) and equations for $\widehat{\widetilde{x}}, x, \widehat{\widetilde{y}}$ in (5.25).

If $b_{0}=0$ we also have the two-component version (2.13c) 2.13d) containing $z$ and $y$ only. Using the CAC method we can construct the $3 \times 3$ Lax matrix

$$
\boldsymbol{L}_{3 \times 3}^{C 3 b_{1}}=\frac{1}{z}\left(\begin{array}{ccc}
\widetilde{z} & 0 & -1 \\
-z \widetilde{y} & z & 0 \\
b_{1} \frac{\widetilde{z}}{y} & -R \frac{z \widetilde{z}}{y} & \frac{1}{y}\left(P z \widetilde{y}-b_{1}\right)
\end{array}\right)
$$

and with corresponding $\boldsymbol{M}_{3 \times 3}^{C 3 b_{1}}$ the compatibility condition exactly returns $(2.13 \mathrm{c}, 2.13 \mathrm{~d})$.

\section{Bilinear structures of DBSQ-type equations}

\subsection{Preliminary}

\subsubsection{Discrete Hirota's bilinear form and Casoratians}

Suppose $f_{j}(n, m)$ and $g_{j}(n, m)$ are functions defined on $\mathbb{Z} \times \mathbb{Z}$. Then a one-component discrete Hirota bilinear equation has the following form [17,20]

$$
\sum_{j} c_{j} f_{j}\left(n+\nu_{j}^{+}, m+\mu_{j}^{+}\right) g_{j}\left(n+\nu_{j}^{-}, m+\mu_{j}^{-}\right)=0,
$$

where it is essential that the index sums $\mu_{j}^{+}+\mu_{j}^{-}=\mu_{s}, \nu_{j}^{+}+\nu_{j}^{-}=\nu_{s}$ do not depend on $j$. 
The solutions to a discrete Hirota bilinear equation will be given by a Casoratian, which is a determinant of a matrix composed of different shifts of a vector. For example, given functions $\psi_{i}(n, m, l)$ we define the column vector

$$
\psi(n, m, l)=\left(\psi_{1}(n, m, l), \psi_{2}(n, m, l), \cdots, \psi_{N}(n, m, l)\right)^{T},
$$

and then the $N$-th order Casoratian reads

$$
\left|\psi\left(n, m, l_{1}\right), \psi\left(n, m, l_{2}\right), \cdots, \psi\left(n, m, l_{N}\right)\right|
$$

For such a determinant we use a shorthand notation $\left|l_{1}, l_{2}, \cdots, l_{N}\right|$. Furthermore, if the Casoratian contains consecutive columns we use condensed notation such as (cf. [10]),

$$
|0,1, \cdots, N-1|=|\widehat{N-1}|, \quad|0,1, \cdots, N-2, N|=|\widehat{N-2}, N| .
$$

For the DBSQ-type equations discussed in this paper the solutions can be expressed through a Casoratian $f=|\widehat{N-1}|$ which is composed by the entry function $\Psi$ :

$$
\begin{aligned}
\psi_{j}(l, n, m, \alpha, \beta):=\sum_{s=0}^{2}\left(-\omega_{s}\left(k_{j}\right)\right)^{l}\left(p-\omega_{s}\left(k_{j}\right)\right)^{n}\left(q-\omega_{s}\left(k_{j}\right)\right)^{m} \\
\times\left(a-\omega_{s}\left(k_{j}\right)\right)^{\alpha}\left(b-\omega_{s}\left(k_{j}\right)\right)^{\beta} \varrho_{j, s}^{(0)},
\end{aligned}
$$

which contains 5 independent variables $n, m, l, \alpha$ and $\beta$. In the following we do not mention those variables that are obvious from context. Here $\omega_{s}\left(k_{j}\right), s=1,2$ are roots of

$$
g_{3}\left(\omega\left(k_{j}\right)\right)=g_{3}\left(k_{j}\right)
$$

where $g_{3}$ defined in (2.29b) and $\omega_{0}\left(k_{j}\right) \equiv k_{j}$.

For shifts in the index variables $(l, n, m, \alpha, \beta)$ we often use shorthand notation: in addition to tilde and hat for shifts in the $n$ - and $m$-direction (as in (2.1)), we introduce bar, circle and dot for the shifts in the $l-, \alpha$ - and $\beta$-directions, respectively, i.e.

$$
\begin{aligned}
\bar{f}(n, m, l, \alpha, \beta) & =f(n, m, l+1, \alpha, \beta), \\
\stackrel{\circ}{f}(n, m, l, \alpha, \beta) & =f(n, m, l, \alpha+1, \beta), \\
\dot{f}(n, m, l, \alpha, \beta) & =f(n, m, l, \alpha, \beta+1) .
\end{aligned}
$$

When the symbol is below the variable it means backward shift: e.g., $\underset{\sim}{f}(n, m, l, \alpha, \beta)=$ $f(n-1, m, l, \alpha, \beta)$.

A more general form than (6.3) is

$$
\psi_{j}(l)=\sum_{s=0}^{2}\left(\delta-\omega_{s}\left(k_{j}\right)\right)^{l}\left(p-\omega_{s}\left(k_{j}\right)\right)^{n}\left(q-\omega_{s}\left(k_{j}\right)\right)^{m}\left(a-\omega_{s}\left(k_{j}\right)\right)^{\alpha}\left(b-\omega_{s}\left(k_{j}\right)\right)^{\beta} \varrho_{j, s}^{(0)},
$$

which is referred to as the $\delta$-extension of (6.3). Thus (6.6) can be considered as a function containing symmetrically five dimensions, corresponding to the direction coordinates $(n, m, l, \alpha, \beta)$ and their lattice spacing parameters $(p, q, a, b, \delta)$. Here for $\psi_{j}(l)$ in (6.6) we already omitted $n, m, \alpha, \beta$ for convenience, we will often do this if it does not cause any confusion. 
Finally we note that a Casoratian can sometimes be written as a Wronskian. Suppose we define

$$
\phi_{j}(l)=\sum_{s=0}^{2} e^{\left(\delta-\omega_{s}\left(k_{j}\right)\right) l}\left(p-\omega_{s}\left(k_{j}\right)\right)^{n}\left(q-\omega_{s}\left(k_{j}\right)\right)^{m}\left(a-\omega_{s}\left(k_{j}\right)\right)^{\alpha}\left(b-\omega_{s}\left(k_{j}\right)\right)^{\beta} \varrho_{j, s}^{(0)},
$$

and the corresponding vector as in (6.1). Then the following Casoratian and Wronskian are equal to each other,

$$
\left|\psi\left(l_{1}\right), \psi\left(l_{2}\right), \cdots, \psi\left(l_{N}\right)\right|_{\mathrm{C}[\psi]}=\left|\partial_{l}^{l_{1}} \phi(l), \partial_{l}^{l_{2}} \phi(l), \cdots, \partial_{l}^{l_{N}} \phi(l)\right|_{\mathrm{w}[\phi]} .
$$

\subsubsection{Laplace expansion}

The proof that a Casoratian solves a bilinear equation is usually given by reducing the problem to a three-term Laplace expansion of a zero determinant. We will first give a generic result.

Lemma 6.1 Suppose that $\mathbf{P}$ is a $N \times(N-1)$ matrix, and $\mathbf{Q}$ its $N \times(N-k+1)$ sub-matrix obtained by removing arbitrary $(k-2)$ columns from $\mathbf{P}$ where $k \geq 3$. Let $\mathbf{a}_{i}(i=1,2, \cdots, k)$ be some $N$-th order column vectors. Then we have

$$
\sum_{i=1}^{k}(-1)^{i-1}\left|\mathbf{P}, \mathbf{a}_{i}\right|\left|\mathbf{Q}, \mathbf{a}_{1}, \cdots, \mathbf{a}_{i-1}, \mathbf{a}_{i+1}, \cdots, \mathbf{a}_{k}\right|=0 .
$$

This is a special case of Plüker relations.

In fact, let $\mathbf{B}$ be the $N \times(k-2)$ matrix consisting of those $(k-2)$ column vectors that are removed from $\mathbf{P}$ so that $|\mathbf{Q B}|=|\mathbf{P}|$. Then it is easy to see that the following $2 N \times 2 N$ determinant vanishes:

$$
\left|\begin{array}{cccccc}
\mathbf{Q} & \mathbf{0} & \mathbf{B} & \mathbf{a}_{1} & \cdots & \mathbf{a}_{k} \\
\mathbf{0} & \mathbf{Q} & \mathbf{0} & \mathbf{a}_{1} & \cdots & \mathbf{a}_{k}
\end{array}\right|=0
$$

The LHS of (6.8) is actually the Laplace expansion of the LHS of (6.9).

Equation (6.8) is quite useful in proving solutions. When $k=3$, (6.8) yields

$$
\left|\mathbf{P}, \mathbf{a}_{1}\right|\left|\mathbf{Q}, \mathbf{a}_{2}, \mathbf{a}_{3}\right|-\left|\mathbf{P}, \mathbf{a}_{2}\right|\left|\mathbf{Q}, \mathbf{a}_{1}, \mathbf{a}_{3}\right|+\left|\mathbf{P}, \mathbf{a}_{3}\right|\left|\mathbf{Q}, \mathbf{a}_{1}, \mathbf{a}_{2}\right|=0 .
$$

If we denote $\mathbf{P}=\left(\mathbf{Q}, \mathbf{a}_{0}\right)$, it reads

$$
\left|\mathbf{Q}, \mathbf{a}_{0}, \mathbf{a}_{1}\right|\left|\mathbf{Q}, \mathbf{a}_{2}, \mathbf{a}_{3}\right|-\left|\mathbf{Q}, \mathbf{a}_{0}, \mathbf{a}_{2}\right|\left|\mathbf{Q}, \mathbf{a}_{1}, \mathbf{a}_{3}\right|+\left|\mathbf{Q}, \mathbf{a}_{0}, \mathbf{a}_{3}\right|\left|\mathbf{Q}, \mathbf{a}_{1}, \mathbf{a}_{2}\right|=0,
$$

which can be viewed as an expression of Plüker relation, and were used to verify Wronskian (or Casoratian) solutions to bilinear equations [10] and also to prove many determinantal identities (see [12]). When $k=4$, (6.8) yields

$$
\begin{aligned}
& \left|\mathbf{P}, \mathbf{a}_{1}\right|\left|\mathbf{Q}, \mathbf{a}_{2}, \mathbf{a}_{3}, \mathbf{a}_{4}\right|-\left|\mathbf{P}, \mathbf{a}_{2}\right|\left|\mathbf{Q}, \mathbf{a}_{1}, \mathbf{a}_{3}, \mathbf{a}_{4}\right|+\left|\mathbf{P}, \mathbf{a}_{3}\right|\left|\mathbf{Q}, \mathbf{a}_{1}, \mathbf{a}_{2}, \mathbf{a}_{4}\right| \\
- & \left|\mathbf{P}, \mathbf{a}_{\mathbf{4}}\right|\left|\mathbf{Q}, \mathbf{a}_{1}, \mathbf{a}_{2}, \mathbf{a}_{3}\right|=0,
\end{aligned}
$$

which is also useful in solution verification (see [43]). In this paper, besides (6.10) and (6.12), we need also $k=5$ case, which is

$$
\begin{aligned}
& \left|\mathbf{P}, \mathbf{a}_{1}\right|\left|\mathbf{Q}, \mathbf{a}_{2}, \mathbf{a}_{3}, \mathbf{a}_{4}, \mathbf{a}_{5}\right|-\left|\mathbf{P}, \mathbf{a}_{2}\right|\left|\mathbf{Q}, \mathbf{a}_{1}, \mathbf{a}_{3}, \mathbf{a}_{4}, \mathbf{a}_{5}\right|+\left|\mathbf{P}, \mathbf{a}_{3}\right|\left|\mathbf{Q}, \mathbf{a}_{1}, \mathbf{a}_{2}, \mathbf{a}_{4}, \mathbf{a}_{5}\right| \\
- & -\mathbf{P}, \mathbf{a}_{4}|| \mathbf{Q}, \mathbf{a}_{1}, \mathbf{a}_{2}, \mathbf{a}_{3}, \mathbf{a}_{5}|+| \mathbf{P}, \mathbf{a}_{5}|| \mathbf{Q}, \mathbf{a}_{1}, \mathbf{a}_{2}, \mathbf{a}_{3}, \mathbf{a}_{4} \mid=0 .
\end{aligned}
$$

Here is another determinantal property which is often used in Wronskian/Casoratian verification. 


\section{Lemma 6.2 [10]}

$$
\sum_{j=1}^{N}\left|\mathbf{a}_{1}, \cdots, \mathbf{a}_{j-1}, \mathbf{b} \mathbf{a}_{j}, \mathbf{a}_{j+1}, \cdots, \mathbf{a}_{N}\right|=\left(\sum_{j=1}^{N} b_{j}\right)\left|\mathbf{a}_{1}, \cdots, \mathbf{a}_{N}\right|,
$$

where $\mathbf{a}_{j}=\left(a_{1 j}, \cdots, a_{N j}\right)^{T}$ and $\mathbf{b}=\left(b_{1}, \cdots, b_{N}\right)^{T}$ are $N$-th order column vectors and $\mathbf{b a}_{j}$ stands for $\left(b_{1} a_{1 j}, \cdots, b_{N} a_{N j}\right)^{T}$.

A generalized version of this lemma can be found in [39,42]. For further details see [40].

\section{$6.2 \quad$ B2}

\subsubsection{B2}

Equation B2 ((2.3) with $\left.b_{1}=0\right)$ is given by

$$
\begin{aligned}
& B_{1}:=\widetilde{y}-x \widetilde{x}+z=0, \\
& B_{2}:=\widehat{y}-x \widehat{x}+z=0, \\
& B_{3}:=y-b_{0}(\widehat{\widetilde{x}}-x)-x \widehat{\widetilde{x}}+\widehat{\widetilde{z}}-\frac{P-Q}{\widetilde{x}-\widehat{x}}=0,
\end{aligned}
$$

where

$$
P=p^{3}-b_{0} p^{2}+R, \quad Q=q^{3}-b_{0} q^{2}+R .
$$

It has background solution 18

$$
\begin{aligned}
& x_{0}=p n+q m+c_{1}, \\
& z_{0}=\frac{1}{2} x_{0}^{2}+\frac{1}{2}\left(p^{2} n+q^{2} m+c_{2}\right)+c_{3}, \\
& y_{0}=\frac{1}{2} x_{0}^{2}-\frac{1}{2}\left(p^{2} n+q^{2} m+c_{2}\right)-c_{3},
\end{aligned}
$$

where $c_{1}, c_{2}, c_{3}$ are arbitrary constants.

By the dependent variable transformation [18

$$
x=x_{0}-\frac{g}{f}, \quad z=z_{0}-x_{0} \frac{g}{f}+\frac{h}{f}, \quad y=y_{0}-x_{0} \frac{g}{f}+\frac{s}{f},
$$

we can bilinearize the B2 lattice consisting of $(6.15)$ and their shifts follows

$$
B_{1}=\frac{\mathcal{B}_{1}}{f \widetilde{f}}, B_{2}=\frac{\mathcal{B}_{2}}{f \widehat{f}}, B_{3}=\frac{\mathcal{B}_{3} \mathcal{B}_{4}+(p-q) f \widehat{\widetilde{f}} \mathcal{B}_{4}+\left[p^{2}+p q+q^{2}-b_{0}(p+q)\right] \tilde{f} \widehat{f} \mathcal{B}_{3}}{(\widetilde{x}-\widehat{x}) f \widetilde{f} \hat{f} \widehat{\widetilde{f}}},
$$

where the bilinear equations are

$$
\begin{aligned}
& \mathcal{B}_{1}:=\widetilde{f}(h+p g)-\widetilde{g}(g+p f)+f \widetilde{s}=0, \\
& \mathcal{B}_{2}:=\widehat{f}(h+q g)-\widehat{g}(g+q f)+f \widehat{s}=0, \\
& \mathcal{B}_{3}:=\widetilde{f} \widehat{g}-\widehat{f} \widetilde{g}+(p-q)(\widetilde{f} \widehat{f}-f \widetilde{\widetilde{f}})=0, \\
& \mathcal{B}_{4}:=\left[p^{2}+p q+q^{2}-b_{0}(p+q)\right](f \widehat{\widetilde{f}}-\widetilde{f} \widehat{f})+\left(p+q-b_{0}\right)(\widehat{\widetilde{f}} g-f \widehat{\widetilde{g}})+\widehat{\widetilde{f}} s+f \widehat{\widetilde{h}}-g \widehat{\widetilde{g}}=0 .
\end{aligned}
$$


We have also used the parametrization (6.15d).

The set of bilinear equations (6.19) admits $N$-soliton solutions in the following Casoratian form,

$$
f=|\widehat{N-1}|, g=|\widehat{N-2}, N|, h=|\widehat{N-2}, N+1|, s=|\widehat{N-3}, N-1, N|,
$$

composed of $\psi=\left(\psi_{1}, \psi_{2}, \cdots, \psi_{N}\right)^{T}$ with (6.3). A proof for these Casoratian solutions can be found in [19], where the meaning of the parameter $b_{0}$ is also discussed.

An alternate bilinearization was given in [22], where they first transformed the 9-point equation (3.10) without $b_{0}$ into a pair of equations living on a $2 \times 4$ stencil (Eqs. $(53,54)$ ), which were then bilinearized using only $f$ and $g$ (see Eqs. $(56,57,65,66)$ ).

\subsubsection{B2- $\delta$}

In the above derivation we used Casoratians with entries (6.3). However, there is a natural generalization of the entry function with a new parameter $\delta$ as given in (6.6). We will now derive the corresponding generalized equations.

As the first step we compute the difference of $f, g, h, s$ (as defined in (6.20) ) for $\delta=0$ and for $\delta \neq 0$. Using binomial expansion on $\left(\delta-\omega_{s}\left(k_{j}\right)\right)^{l}$ we see that only the right-most columns contribute and find [18] (here $f \equiv f(0), f^{\prime} \equiv f(\delta)$ )

$$
\begin{aligned}
f^{\prime} & =f, \\
g^{\prime} & =g+N \delta f, \\
h^{\prime} & =h+(N+1) \delta g+\frac{1}{2} N(N+1) \delta^{2} f, \\
s^{\prime} & =s+(N-1) \delta g+\frac{1}{2} N(N-1) \delta^{2} f,
\end{aligned}
$$

where $N$ is the size of the determinant. Now inverting this for $f, g, h, s$ and using them on (6.19) it follows that the primed quantities solve the $\delta$-modified bilinear equations

$$
\begin{aligned}
& \mathcal{B}_{1}^{\delta}:=\widetilde{f^{\prime}}\left[h^{\prime}+(p-\delta) g^{\prime}\right]-\widetilde{g^{\prime}}\left[g^{\prime}+(p-\delta) f^{\prime}\right]+f^{\prime} \widetilde{s^{\prime}}=0, \\
& \mathcal{B}_{2}^{\delta}:=\widehat{f^{\prime}}\left[h^{\prime}+(q-\delta) g^{\prime}\right]-\widehat{g^{\prime}}\left[g^{\prime}+(q-\delta) f^{\prime}\right]+f^{\prime} \widehat{s^{\prime}}=0, \\
& \mathcal{B}_{3}^{\delta}:=\widetilde{f}^{\prime} \widehat{g^{\prime}}-\widehat{f^{\prime}} \widetilde{g^{\prime}}+(p-q)\left(\widetilde{f^{\prime}} \widehat{f}^{\prime}-f^{\prime} \widehat{\tilde{f}^{\prime}}\right)=0, \\
& \mathcal{B}_{4}^{\delta}:=\left(p^{2}+p q+q^{2}-b_{0}(p+q)\right)\left(f^{\prime} \widehat{\widetilde{f}}^{\prime}-\widetilde{f}^{\prime} \widehat{f}^{\prime}\right) \\
& -\left(p+q-b_{0}+\delta\right)\left(f^{\prime} \widehat{\widetilde{g^{\prime}}}-\widehat{\tilde{f}^{\prime}} g^{\prime}\right)+\widehat{\widetilde{f^{\prime}}} s^{\prime}+f^{\prime} \widehat{\widetilde{h^{\prime}}}-g^{\prime} \widehat{\widetilde{g^{\prime}}}=0 .
\end{aligned}
$$

Furthermore, from (6.17) we get

$$
x^{\prime}=x_{0}^{\prime}-\frac{g^{\prime}}{f^{\prime}}=\left(x_{0}^{\prime}-N \delta\right)-\frac{g}{f}=x,
$$

provided that $x_{0}^{\prime}=x_{0}+N \delta$, which can be accommodated by a change in the constant: $c_{1}^{\prime}=c_{1}+N \delta$. Similarly

$$
\begin{aligned}
z^{\prime} & =z_{0}^{\prime}-x_{0}^{\prime} \frac{g^{\prime}}{f^{\prime}}+\frac{h^{\prime}}{f^{\prime}} \\
& =z_{0}^{\prime}-\left(x_{0}+N \delta\right) \frac{g}{f}-x_{0}^{\prime} N \delta+\frac{h}{f}+(N+1) \delta \frac{g}{f}+\frac{1}{2} N(N+1) \delta^{2} \\
& =z-\delta\left(x-x_{0}\right), \\
y^{\prime} & =y+\delta\left(x-x_{0}\right),
\end{aligned}
$$


provided that the constants in $z_{0}, y_{0}$ are adjusted so that $\frac{1}{2}\left(c_{2}^{\prime}-c_{2}\right)+c_{3}^{\prime}-c_{3}+\frac{1}{2} \delta^{2} N=0$. Using these we get the $\delta$-modified nonlinear equations:

$$
\begin{aligned}
& B_{1}^{\delta}:=\widetilde{y^{\prime}}-x^{\prime} \widetilde{x^{\prime}}+z^{\prime}-\delta\left(\widetilde{x^{\prime}}-x^{\prime}-p\right)=0, \\
& B_{2}^{\delta}:=\widehat{y^{\prime}}-x^{\prime} \widehat{x^{\prime}}+z^{\prime}-\delta\left(\widehat{x^{\prime}}-x^{\prime}-q\right)=0, \\
& B_{3}^{\delta}:=y^{\prime}-b_{0}\left(\widehat{\widetilde{x^{\prime}}}-x^{\prime}\right)-x^{\prime} \widehat{\widetilde{x^{\prime}}}+\widehat{\widetilde{z^{\prime}}}+\frac{p^{3}-q^{3}-b_{0}\left(p^{2}-q^{2}\right)}{\widehat{x^{\prime}}-\widetilde{x^{\prime}}}+\delta\left(\widehat{x^{\prime}}-x^{\prime}-p-q\right)=0 .
\end{aligned}
$$

Equations (6.22) and (6.24) were given for the $b_{0}=0$ case in [18] and for generic $b_{0}$ in [32].

\section{$6.3 \quad \mathrm{~A} 2$}

\subsection{1 $\mathrm{A} 2$}

The A2 equation (after removing parameter $b_{0}$ ) is given by

$$
\begin{aligned}
& A_{1}:=\widetilde{x} z-\widetilde{y}-x=0, \\
& A_{2}:=\widehat{x} z-\widehat{y}-x=0, \\
& A_{3}:=x \widehat{\widetilde{z}}-y-\frac{P \widetilde{x}-Q \widehat{x}}{\widetilde{z}-\widehat{z}}=0,
\end{aligned}
$$

where $P, Q$ will be parameterized by (2.37). It has background or seed solution (2.36)

$$
\begin{aligned}
& x_{a}=(p-a)^{-n}(q-a)^{-m} c_{1}, \\
& z_{0}=\left(c_{3}-p\right) n+\left(c_{3}-q\right) m+c_{2}, \\
& y_{a}=x_{a}\left(z_{0}+a-c_{3}\right),
\end{aligned}
$$

where $c_{3}=\alpha_{2} / 3$, and $c_{1}, c_{2}, \alpha_{i}$ are arbitrary constants.

By the dependent variable transformation

$$
x=x_{a} \frac{f}{f}, \quad z=z_{0}+\frac{g}{f}, \quad y=y_{a} \frac{f}{f}+x_{a} \frac{g}{f},
$$

A2 is bilinearized into

$$
\begin{aligned}
& \mathcal{A}_{1}:=\widetilde{f}(g+p f)-(p-a) \underset{0}{f} \tilde{f}-f(a \tilde{f}+\widetilde{g})=0, \\
& \mathcal{A}_{2}:=\widehat{f}(g+q f)-(q-a) f \widehat{f}-f(a \widehat{f}+\widehat{g})=0, \\
& \mathcal{A}_{3}:=\tilde{f} \widehat{g}-\widehat{f} \widetilde{g}+(p-q)(\tilde{f} \widehat{f}-f \widehat{\widetilde{f}})=0,
\end{aligned}
$$

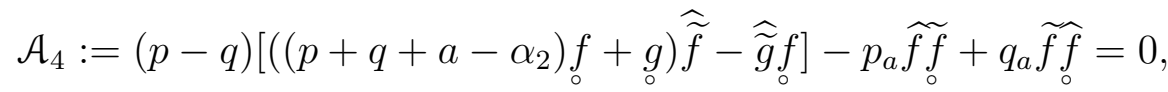

where $p_{a}, q_{a}$ are defined as (2.34) and the circle shift was defined in (6.5). The connection to (6.25) is by

$$
A_{1}=\frac{\widetilde{x}_{a} \mathcal{A}_{1}}{f \widetilde{f}}, A_{2}=\frac{\widehat{x}_{a} \mathcal{A}_{2}}{f \widehat{f}}, A_{3}=x_{a} \frac{\mathcal{A}_{3} \mathcal{A}_{4}+(p-q) f \widehat{\widetilde{f}} \mathcal{A}_{4}+\left(p_{a} \widehat{f f} q_{0}-q_{a} \widetilde{f} \widehat{f}\right) \mathcal{A}_{3}}{(p-q) f \widetilde{f} \widehat{f} \widehat{\widetilde{f}}(\widehat{z}-\widetilde{z})} .
$$

Multisoliton solutions to (6.28) are given through

$$
f=|\widehat{N-1}|, g=|\widehat{N-2}, N|,
$$

composed of $\psi$ given in (6.6) with $\delta=0$. 


\subsection{2 $\mathrm{A} 2-\delta$}

The $\delta$ deformation discussed in Section 6.2.2 can also be applied on A2. In this case the definitions of $f, g$ are as in (6.20), and therefore the $\delta$-dependence is as in (6.21), i.e., $f^{\prime}=f, g^{\prime}=g+N \delta f$. From this and (6.27) it follows

$$
x=x^{\prime}, \quad y=y^{\prime}, \quad z=z^{\prime}-\delta,
$$

provided that we choose $c_{2}^{\prime}-c_{2}+N \delta=0$. Then we find A2- $\delta$ as

$$
\begin{aligned}
& A_{1}^{\delta}:=\widetilde{x^{\prime}} z^{\prime}-\widetilde{y^{\prime}}-x^{\prime}-\delta \widetilde{x^{\prime}}=0, \\
& A_{2}^{\delta}:=\widehat{x^{\prime}} z^{\prime}-\widehat{y^{\prime}}-x^{\prime}-\delta \widehat{x^{\prime}}=0, \\
& A_{3}^{\delta}:=x^{\prime} \widetilde{\widetilde{z}}^{\prime}-y^{\prime}-\delta x^{\prime}+\frac{P \widetilde{x^{\prime}}-Q \widehat{x^{\prime}}}{\widetilde{z^{\prime}}-\widehat{z^{\prime}}}=0 .
\end{aligned}
$$

On the other hand, it can be easily seen that equations (6.28) are invariant under $g \mapsto$ $g+c f$.

\section{4 $\quad \mathrm{C3}_{b_{0}}$}

The equation $\mathrm{C} 3_{b_{0}}$ is

$$
\begin{aligned}
& C_{1}:=\widetilde{x}-\widetilde{y} z-x=0, \\
& C_{2}:=\widehat{x}-\widehat{y} z-x=0, \\
& C_{3}:=\widehat{\widetilde{z}} y-d_{2} x-z \frac{P \widetilde{y} \widehat{z}-Q \widehat{y} \widetilde{z}}{\widetilde{z}-\widehat{z}}=0 .
\end{aligned}
$$

It has 0SS

$$
\begin{aligned}
& x_{a, b}=\frac{1}{b-a}\left(\frac{p-b}{p-a}\right)^{n}\left(\frac{p-b}{q-a}\right)^{m}, \\
& z_{b}=(p-b)^{n}(q-b)^{m} \\
& y_{a}=-(p-a)^{-n}(q-a)^{-m}
\end{aligned}
$$

where we have used parametrization (2.46). By the transformation (c.f., (6.5))

$$
x=x_{a, b} \frac{\dot{\circ}}{f}, \quad z=z_{b} \frac{\dot{f}}{f}, \quad y=y_{a} \frac{f}{f},
$$

from (6.33) we have its bilinear form

$$
\begin{aligned}
& \mathcal{C}_{1}:=(p-b) \underset{\tilde{f}_{0}}{\dot{\tilde{f}}} f-(p-a) \underset{\mathrm{f}}{\dot{f} \tilde{f}}+(b-a) \underset{f}{\tilde{f} \tilde{f}}=0, \\
& \mathrm{C}_{2}:=(q-b) \underset{\mathrm{f}}{\hat{\mathrm{f}}} f-(q-a) \dot{\mathrm{f}} \widehat{\mathrm{f}}+(b-a) \dot{\mathrm{f}} \widehat{\mathrm{f}}=0, \\
& \mathrm{C}_{3}:=(p-b) \hat{f} \dot{\widetilde{f}}-(q-b) \tilde{f} \dot{\hat{f}}+(p-q) \hat{\widetilde{f}} \dot{f}=0,
\end{aligned}
$$

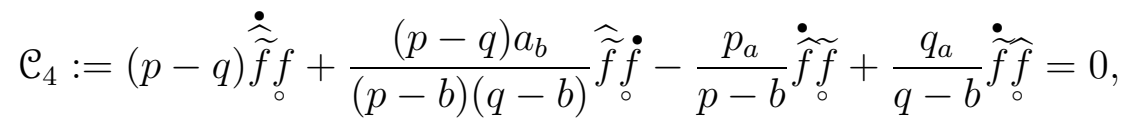


where $p_{a}, q_{a}$ are defined as (2.34) and here we also use $a_{b}=b_{a}=\left.p_{a}\right|_{p=b}$, and the connection with (6.33) is

$$
\begin{aligned}
C_{1} & =\frac{-x_{a, b} \mathcal{C}_{1}}{(p-a) f \widetilde{f}}, \quad C_{2}=\frac{-x_{a, b} \mathcal{C}_{2}}{(q-a) f \widehat{f}}, \\
C_{3} & =\frac{(p-b)(q-b)(b-a) x_{a, b} z_{b}}{(p-q) f \widetilde{f} \widehat{f} \widehat{\tilde{f}}(\widetilde{z}-\widehat{z})}\left[\mathcal{C}_{3} \mathcal{C}_{4}+\left(\frac{p_{a}}{p-b} \dot{\widetilde{f}} \widetilde{\circ}-\frac{q_{a}}{q-b} \dot{\widetilde{f}} \widehat{f}\right) \mathcal{C}_{3}-(p-q) \hat{\widetilde{f}} \dot{f} \mathcal{C}_{4}\right] .
\end{aligned}
$$

Casoratian solution of $(\underline{6.36})$ is given by $f=|\widehat{N-1}|$ with $\psi$ composed by (ㅎ.6). Note that $\mathrm{C} 3-\delta$ is the same as (6.33).

The bilinearization of $\mathrm{C} 3_{b_{1}}$ is still open.

\section{Conclusions}

In this review we have discussed the fully discrete versions Boussinesq equations given as three-component equations on the basic quadrilateral. Their derivation using CAC and DLA was compared. From the three-component equation we derived two- and onecomponent versions on a larger stencil. Then we derived two semi-continuous limits and the fully continuous limits for the one-component versions. We discussed also several versions of Lax pairs. Finally we gave their Hirota bilinear forms, which are important for constructing solutions. The basic results are summarized in the adjoining Table 1 which points to the relevant equations.

We also note that recently an elliptic scheme of DLA has been developed, in which the spacing parameters $P$ and $Q$ can be parameterized by Weierstrass elliptic functions [29].

In addition to the question of bilinearizing equation $\mathrm{C} 3_{b_{1}}$, there are some interesting open questions: For example, the classification of higher order CAC lattice equations (cf. the ABS list containing 9 equations), higher genus solutions of the DBSQ-type equations, reductions of the hierarchy of lattice KP equations, etc.

\section{Acknowledgments}

We would like to thank J. Schiff for bringing equation (3.9) to our attention. This work was supported by the NSF of China (grant numbers 11875040 and 11631007). All computations were done with REDUCE [13].

\section{Appendix A Triply shifted variables from CAC}

Since the equations are integrable their triply shifted forms are the same independent of the sides used in the computation, therefore tilde-hat-bar symmetric. They can be computed if the equations are defined on a quadrilateral. This holds for all three-component forms and for some two-component forms but the one component forms are all defined on a bigger stencil. 


\begin{tabular}{|c|c|c|c|c|}
\hline Equation name & $\mathrm{A} 2$ & $\mathrm{~B} 2$ & $\mathrm{C} 3_{b_{0}}$ & $\mathrm{C} 3_{b_{1}}$ \\
\hline 3 component versions & $(2.4)$, , (2.24) & $(2.3)$ & 2.12 & $\leftarrow$ \\
\hline 2 component versions & $(3.2),(3.3)$ & (3.8) & (3.12), (3.18) & (3.14) \\
\hline 1 component versions & (3.5), (3.6) & $(3.10)$ & (3.13), (3.5) & $\leftarrow$ \\
\hline Straight limit & (4.19) & 4.11 & (4.24) & $\leftarrow$ \\
\hline Skew limit & 4.21 & 4.12 & 4.25 & $\leftarrow$ \\
\hline Double limit & 4.22 & 4.16 & 4.26 & $\leftarrow$ \\
\hline Lax Pairs & (5.15) +others & $(5.8),(5.11)$ & $(5.26)$ & 5.28 \\
\hline Bilinear form & (6.28) & (6.19) & (6.36) & $?$ \\
\hline
\end{tabular}

Table 1: A summary of the relevant equations. Those defining equations that live on the elementary quadrilateral are boxed. The " $\leftarrow$ " in the last column means the result is included in the $\mathrm{C}_{b_{0}}$ case.

\section{A.1 Three-component forms}

Three-component forms all live in the elementary quadrilateral and were in fact derived using CAC. The triply shifted quantities are as follows [14]:

For A2

$$
\begin{aligned}
& \overline{\widehat{\widetilde{x}}}=x \frac{\widetilde{x}(\widehat{z}-\bar{z})+\widehat{x}(\bar{z}-\widetilde{z})+\bar{x}(\widetilde{z}-\widehat{z})}{p \widetilde{x}(\widehat{z}-\bar{z})+q \widehat{x}(\bar{z}-\widetilde{z})+r \bar{x}(\widetilde{z}-\widehat{z})}, \\
& \overline{\widehat{\widetilde{y}}}=\frac{y}{x} \widehat{\widehat{\widetilde{x}}}+\frac{p \widetilde{x}(\widehat{x}-\bar{x})+q \widehat{x}(\bar{x}-\widetilde{x})+r \bar{x}(\widetilde{x}-\widehat{x})}{p \widetilde{x}(\widehat{z}-\bar{z})+q \widehat{x}(\bar{z}-\widetilde{z})+r \bar{x}(\widetilde{z}-\widehat{z})}, \\
& \overline{\widehat{\widetilde{z}}}=z-x \frac{p(\widehat{z}-\bar{z})+q(\bar{z}-\widetilde{z})+r(\widetilde{z}-\widehat{z})}{p \widetilde{x}(\widehat{z}-\bar{z})+q \widehat{x}(\bar{z}-\widetilde{z})+r \bar{x}(\widetilde{z}-\widehat{z})} .
\end{aligned}
$$

For B2

$$
\begin{aligned}
& \overline{\widehat{\widetilde{x}}}=b_{0}+x+\frac{(q-r) \widetilde{x}+(r-p) \widehat{x}+(p-q) \bar{x}}{\widetilde{x}(\widehat{z}-\bar{z})+\widehat{x}(\bar{z}-\widetilde{z})+\bar{x}(\widetilde{z}-\widehat{z})}, \\
& \overline{\widehat{\widetilde{y}}}=b_{0} x+y+\frac{(q-r) \widetilde{z}+(r-p) \widehat{z}+(p-q) \bar{z}}{\widetilde{x}(\widehat{z}-\bar{z})+\widehat{x}(\bar{z}-\widetilde{z})+\bar{x}(\widetilde{z}-\widehat{z})}, \\
& \overline{\widehat{\widetilde{z}}}=z+b_{0} \overline{\widehat{\widetilde{x}}}-\frac{(q-r) \widehat{x} \bar{x}+(r-p) \bar{x}+(p-q) \widetilde{x} \widehat{x}}{\widetilde{x}(\widehat{z}-\bar{z})+\widehat{x}(\bar{z}-\widetilde{z})+\bar{x}(\widetilde{z}-\widehat{z})} .
\end{aligned}
$$

For C3

$$
\begin{aligned}
\overline{\widehat{\widetilde{x}}}= & \frac{b_{0} x+b_{1}}{y} \overline{\widehat{\widetilde{y}}}+x+z \frac{\widetilde{z} \widehat{y} \bar{y}(q-r)+\widehat{z} \bar{y} \widetilde{y}(r-p)+\bar{z} \widetilde{y} \widehat{y}(p-q)}{\widetilde{y}(q \bar{y})+\widehat{z}(r \bar{y}-p \widetilde{y})+\bar{z}(p \widetilde{y}-q \widehat{y})}, \\
\overline{\widehat{\widetilde{y}}}= & y \frac{\widetilde{z}(\widehat{y}-\bar{y})+\widehat{z}(\bar{y}-\widetilde{y})+\bar{z}(\widetilde{y}-\bar{y})}{\widetilde{z}(q \widehat{y}-r \bar{y})+\widehat{z}(r \bar{y}-p \widetilde{y})+\bar{z}(p \widetilde{y}-q \widehat{y})}, \\
\overline{\widehat{\widetilde{z}}}= & b_{0} z+\left(b_{1}+b_{0} x\right) \frac{(q-r) \widetilde{z}+(r-p) \widehat{z}+(p-q) \bar{z}}{\widetilde{z}(q \widehat{y}-r \bar{y})+\widehat{z}(r \bar{y}-p \widetilde{y})+\bar{z}(p \widetilde{y}-q \widehat{y})} \\
& +z y \frac{q r \widetilde{z}(\widehat{y}-\bar{y})+r p \widehat{z}(\bar{y}-\widetilde{y})+p q \bar{z}(\widetilde{y}-\bar{y})}{\widetilde{z}(q \widehat{y}-r \bar{y})+\widehat{z}(r \bar{y}-p \widetilde{y})+\bar{z}(p \widetilde{y}-q \widehat{y})} .
\end{aligned}
$$




\section{A.2 Two-component forms}

When the two component form is defined on a quadrilateral we can compute the triply shifted quantities. This is true for $(3.3),(3.14),(3.18)$.

For A2 (3.3) where $w:=y / x$

$$
\begin{aligned}
\overline{\widehat{\widetilde{w}}}= & w+\frac{P(\bar{w}-\widehat{w})+Q(\widetilde{w}-\bar{w})+R(\widehat{w}-\widetilde{w})}{\widetilde{z}(\widetilde{w}-z)(\widehat{w}-\bar{w})+\widehat{z}(\widehat{w}-z)(\bar{w}-\widetilde{w})+\bar{z}(\bar{w}-z)(\widetilde{w}-\widehat{w})}, \\
\overline{\widetilde{z}}= & \\
& \frac{P \widetilde{w}(\widehat{w}-z)(\bar{w}-z)(\widehat{z}-\bar{z})+Q \widehat{w}(\bar{w}-z)(\widetilde{w}-z)(\bar{z}-\widetilde{z})+R \bar{w}(\widetilde{w}-z)(\widehat{w}-z)(\widetilde{z}-\widehat{z})}{P(\widehat{w}-z)(\bar{w}-z)(\widehat{z}-\bar{z})+Q(\bar{w}-z)(\widetilde{w}-z)(\bar{z}-\widetilde{z})+R(\widetilde{w}-z)(\widehat{w}-z)(\widetilde{z}-\widehat{z})} .
\end{aligned}
$$

For B2 (3.9) where $w:=y+z$

$$
\begin{aligned}
\overline{\widehat{x}}= & b_{0}+\frac{P(\bar{x}-\widehat{x})+Q(\widetilde{x}-\bar{x})+R(\widehat{x}-\widetilde{x})}{\widetilde{w}(\bar{x}-\widehat{x})+\widehat{w}(\widetilde{x}-\bar{x})+\bar{w}(\widehat{x}-\widetilde{x})} \\
\overline{\widehat{\widetilde{w}}}= & \overline{\widetilde{x}}\left(b_{0}-x\right)+w+x^{2}+ \\
& \frac{P(\bar{w}-\widehat{w}+(\bar{x}-\widehat{x}) \widetilde{x})+Q(\widetilde{w}-\bar{w}+(\widetilde{x}-\bar{x}) \widehat{x})+R(\widehat{w}-\widetilde{w}+(\widehat{x}-\widetilde{x}) \bar{x})}{\widetilde{w}(\bar{x}-\widehat{x})+\widehat{w}(\widetilde{x}-\bar{x})+\bar{w}(\widehat{x}-\widetilde{x})} .
\end{aligned}
$$

For $\mathrm{C} 3_{b_{1}}(3.14)$ :

$$
\begin{aligned}
\overline{\widetilde{y}}= & -y \frac{\widetilde{y}(\widehat{z}-\bar{z})+\widehat{y}(\bar{z}-\widetilde{z})+\bar{y}(\widetilde{z}-\widehat{z})}{P \widetilde{y}(\widehat{z}-\bar{z})+Q \widehat{y}(\bar{z}-\widetilde{z})+R \bar{y}(\widetilde{z}-\widehat{z})}, \\
\overline{\widehat{z}}= & -z \frac{P Q \bar{z}(\widetilde{y}-\widehat{y})+R P \widehat{z}(\bar{y}-\widetilde{y})+Q R \widetilde{z}(\widehat{y}-\bar{y})}{P \widetilde{y}(\widehat{z}-\bar{z})+Q \widehat{y}(\bar{z}-\widetilde{z})+R \bar{y}(\widetilde{z}-\widehat{z})} \\
& +b_{1} \frac{P(\widehat{z}-\bar{z})+Q(\bar{z}-\widetilde{z})+R(\widetilde{z}-\widehat{z})}{P \widetilde{y}(\widehat{z}-\bar{z})+Q \widehat{y}(\bar{z}-\widetilde{z})+R \bar{y}(\widetilde{z}-\widehat{z})} .
\end{aligned}
$$

For $\mathrm{C} 3_{b_{0}}(3.18)$ :

$$
\begin{aligned}
& \overline{\widehat{\widetilde{w}}}=-w \frac{(\widetilde{w} \widehat{w} z+\bar{w})(\widetilde{z}-\widehat{z})+(\widehat{w} \bar{w} z+\widetilde{w})(\widehat{z}-\bar{z})+(\bar{w} \widetilde{w} z+\widehat{w})(\bar{z}-\widetilde{z})}{\mathcal{D}_{w}}, \\
& \mathcal{D}_{w}=\widetilde{w}\left(P+b_{0}\right)(z(\widehat{w} \widehat{z}-\overline{w z})-\widehat{z}+\bar{z})+\widehat{w}\left(Q+b_{0}\right)(z(\overline{w z}-\widetilde{w} \widetilde{z})-\bar{z}+\widetilde{z}) \\
& +\bar{w}\left(R+b_{0}\right)(z(\widetilde{w} \widetilde{z}-\widehat{w} \widehat{z})-\widetilde{z}+\widehat{z}) \\
& \overline{\widetilde{z}}=z \frac{\mathcal{N}_{z}}{\mathcal{D}_{z}}, \\
& \mathcal{N}_{z}=P Q(\widetilde{w}-\widehat{w})(\bar{w} z-1) \bar{z}+Q R(\widehat{w}-\bar{w})(\widetilde{w} z-1) \widetilde{z}+R P(\bar{w}-\widetilde{w})(\widehat{w} z-1) \widehat{z} \\
& +b_{0}[P(\widehat{w} z-1)(\bar{w} z-1)(\widehat{z}-\bar{z})+Q(\bar{w} z-1)(\widetilde{w} z-1)(\bar{z}-\widetilde{z}) \\
& +R(\widetilde{w} z-1)(\widehat{w} z-1)(\widetilde{z}-\widehat{z})], \\
& \mathcal{D}_{z}=P(\widehat{w} z-1)(\bar{w} z-1)(\widehat{z}-\bar{z}) \widetilde{w}+Q(\bar{w} z-1)(\widetilde{w} z-1)(\bar{z}-\widetilde{z}) \widehat{w} \\
& +R(\widetilde{w} z-1)(\widehat{w} z-1)(\widetilde{z}-\widehat{z}) \bar{w} \text {. }
\end{aligned}
$$

Note that none of these results have the tetrahedron property. 


\section{References}

[1] Adler V E, Bobenko A I and Suris Yu B, Classification of integrable equations on quad-graphs. The consistency approach, Commun. Math. Phys. 233, 513-543, 2003.

[2] Bobenko A I and Suris Yu B, Integrable systems on quad-graphs, Int. Math. Res. Not. 11, 573-611, 2002.

[3] Bridgman T J, LaxPairPartialDifferenceEquations.m: A Mathematica package for the symbolic computation of Lax pairs of nonlinear partial difference equations defined on quadrilaterals. http://inside.mines.edu/ whereman/software/ LaxPairPartialDifferenceEquations/V2, 2017.

[4] Bridgman T, Hereman W, Quispel G R W and van der Kamp P H, Symbolic computation of Lax pairs of partial difference equations using consistency around the cube, Found. Comput. Math. 13, 517-544, 2013.

[5] Bridgman T and Hereman W, Lax pairs for edge-constrained Boussinesq systems of partial difference equations, in Nonlinear Systems and Their Remarkable Mathematical Structures, Vol.2, Eds. N. Euler, M.C. Nucci, Taylor \& Francis, 2019. Boca Raton, FL 33487, U.S.A. pp. 60-88.

[6] Date E, Jimbo M and Miwa T, Method for generating discrete soliton equations. III J. Phys. Soc. Jpn. 52 388-393, 1983.

[7] Feng W, Zhao S L and Zhang D J, Exact solutions to lattice Boussinesq-type equations, J. Nonlin. Math. Phys. 19, No.1250032 (15pp), 2012.

[8] Fokas A S and Ablowitz M J, Linearization of the Korteweg-de Vries and Painlevé II equations. Phys. Rev. Lett. 47, 1096-1110, 1981.

[9] Fordy $\mathrm{A}$ and Xenitidis $\mathrm{P}, \mathbb{Z}_{N}$ graded discrete Lax pairs and integrable difference equations, J. Phys. A: Math. Theor. 50, No.165205 (30pp), 2017.

[10] Freeman N C and Nimmo J J C, Soliton solutions of the KdV and KP equations: the Wronskian technique, Phys. Lett. A 95, 1-3, 1983.

[11] Fu W and Nijhoff F W, Direct linearizing transform for three-dimensional discrete integrable systems: the lattice AKP, BKP and CKP equations, Proc. R. Soc. A 473, No.20160915 (22pp), 2017.

[12] Gragg W B, The Padé table and its relation to certain algorithms of numerical analysis, SIAM Review 14, 1-62, 1972.

[13] Hearn A C and Schöpf R, REDUCE User Manual

https://reduce-algebra.sourceforge.io/, 2019.

[14] Hietarinta J, Boussinesq-like multi-component lattice equations and multidimensional consistency, J. Phys. A: Math. Theor. 44, No.165204 (22pp), 2011.

[15] Hietarinta J, Search for CAC-integrable homogeneous quadratic triplets of quad equations and their classification by BT and Lax, J. Nonlin. Math. Phys. 26, 358-389, 2019 . 
[16] Hietarinta J, Joshi N and Nijhoff F W, Discrete Systems and Integrability, Camb. Univ. Press, Cambridge, 2016.

[17] Hietarinta J and Zhang D J, Soliton solutions for ABS lattice equations: II: Casoratians and bilinearization, J. Phys. A: Math. Theor. 42, No.404006 (30pp), 2009.

[18] Hietarinta J and Zhang D J, Multisoliton solutions to the lattice Boussinesq equation, J. Math. Phys. 51, No.033505 (12pp), 2010.

[19] Hietarinta J and Zhang D J, Soliton taxonomy for a modification of the lattice Boussinesq equation, SIGMA 7, No.061 (14pp), 2011.

[20] Hietarinta J and Zhang D J, Hirota's method and the search for integrable partial difference equations. 1. Equations on a $3 \times 3$ stencil, J. Difference Equa. Appl. 19, 1292-1316, 2013.

[21] Hirota R, and Satsuma J, Nonlinear evolution equations generated from the Bäcklund transformation for the Boussinesq equation, Prog. Theore. Phys. 57, 797-807, 1977.

[22] Maruno K, and Kajiwara K, The discrete potential Boussinesq equation and its multisoliton solutions, Applicable Analysis 89, 593-609, 2010.

[23] Nijhoff F W, On some "Schwarzian Equations" and their discrete analogues, in: Eds. A.S. Fokas and I.M. Gel'fand, Algebraic Aspects of Integrable Systems: In memory of Irene Dorfman, Birkhäuser Boston, 237-260, 1997.

[24] Nijhoff F W, Discrete Painlevé equations and symmetry reduction on the lattice, in: Eds. A.I. Bobenko and R. Seiler, Discrete Integrable Geometry and Physics, Oxford Univ. Press, 209-234, 1999.

[25] Nijhoff F W, Lax pair for the Adler (lattice Krichever-Novikov) system, Phys. Lett. A 297, 49-58, 2002.

[26] Nijhoff F W and Capel H W, The discrete Korteweg-de Vries equation, Acta Appl. Math. 39, 133-158, 1995.

[27] Nijhoff F W, Papageorgiou V G, Capel H W and Quispel G R W, The lattice Gel'fand-Dikii hierarchy, Inverse Problems 8, 597-621, 1992.

[28] Nijhoff F W, Quispel G R W and Capel H W, Direct linearization of nonlinear difference-difference equations, Phys. Lett. A 97, 125-128, 1983.

[29] Nijhoff F W, Sun Y Y and Zhang D J, Elliptic solutions of Boussinesq type lattice equations and the elliptic Nth root of unity, arXiv: 1909.02948.

[30] Nijhoff F W and Walker A J, The discrete and continuous Painlevé VI hierarchy and the Garnier systems, Glasgow Math. J. 43A, 109-123, 2001.

[31] Nong L J, Solutions to Discrete Boussinesq-type System, PhD Thesis, Shanghai Univ., 2014.

[32] Nong L J, Zhang D J, Shi Y and Zhang W Y, Parameter extension and the quasirational solution of a lattice Boussinesq equation, Chin. Phys. Lett. 30, No.040201 (4pp), 2013. 
[33] Quispel G R W, Nijhoff F W, Capel H W and van der Linden J, Linear integral equations and nonlinear difference-difference equations, Physica 125A, 344-380, 1984.

[34] Rasin A G and Schiff J, Bäcklund transformations for the Boussinesq equation and merging solitons, J. Phys. A: Math. Theor. 50, No.325202 (21pp), 2017.

[35] Seiler W M, Involution, Springer, 2010.

[36] Tongas A S and Nijhoff F W, The Boussinesq integrable system. Compatible lattice and continuum structures, Glasgow Math. J. 47A, 205-219, 2005.

[37] Walker A J, Similarity Reductions and Integrable Lattice Equations, PhD Thesis, Univ. Leeds, 2001.

[38] Weiss J, The Painlevé property for partial differential equations. II: Bäcklund transformation, Lax pairs, and the Schwarzian derivative, J. Math. Phys. 24, 1405-1413, 1983.

[39] Zhang D J, Notes on solutions in Wronskian form to soliton equations: KdV-type, arXiv:nlin/0603008v3 [nlin.SI].

[40] Zhang D J, Wronskian solutions of integrable systems, in Nonlinear Systems and Their Remarkable Mathematical Structures, Vol.2, Eds. N. Euler, M.C. Nucci, Taylor \& Francis, 2019. Boca Raton, FL 33487, U.S.A. pp. 415-444. arXiv: 1903.09283.

[41] Zhang D J, Zhao S L and Nijhoff F W, Direct linearization of an extended lattice BSQ system, Stud. Appl. Math. 129, 220-248, 2012.

[42] Zhang D J, Zhao S L, Sun Y Y and Zhou J, Solutions to the modified Korteweg-de Vries equation, Rev. Math. Phys. 26, No.14300064 (42pp), 2014.

[43] Zhou J, Zhang D J and Zhao S L, Breathers and limit solutions of the nonlinear lumped self-dual network equation, Phys. Lett. A 373, 3248-3258, 2009. 